CERN-TH/97-180

July 1997

\title{
Flat Symplectic Bundles of $N$-Extended Supergravities, Central Charges and Black-Hole Entropy $\mathrm{Q}$
}

\author{
Laura Andrianopoli ${ }^{a}$, Riccardo D'Auria ${ }^{b}$ and Sergio Ferrara ${ }^{c}$ \\ ${ }^{a}$ Dipartimento di Fisica, Universitá di Genova, via Dodecaneso 33, I-16146 Genova \\ and Istituto Nazionale di Fisica Nucleare (INFN) - Sezione di Torino, Italy \\ ${ }^{b}$ Dipartimento di Fisica, Politecnico di Torino, \\ Corso Duca degli Abruzzi 24, I-10129 Torino \\ and Istituto Nazionale di Fisica Nucleare (INFN) - Sezione di Torino, Italy \\ ${ }^{c}$ CERN Theoretical Division, CH 1211 Geneva 23, Switzerland

\section{Summary}

In these lectures we give a geometrical formulation of $N$-extended supergravities which generalizes $N=2$ special geometry of $N=2$ theories.

In all these theories duality symmetries are related to the notion of "flat symplectic bundles" and central charges may be defined as "sections" over these bundles. Attractor points giving rise to "fixed scalars" of the horizon geometry and Bekenstein-Hawking entropy formula for extremal black-holes are discussed in some details.

\section{Introduction}

Recent developments on duality symmetries [1] in supersymmetric quantum theories of fields and strings seem to indicate that the known different string theories are different manifestations, in different regions of the coupling constant space, of a unique more fundamental theory that, depending on the regime and on the particular compactification, may itself reveal extra (11 or 12) dimensions [2], [3]. A basic aspect that allows a comparison of different theories is their number of supersymmetries and their spectrum of massless and massive BPS states. Indeed, to explore a theory in the nonperturbative regime, the power of supersymmetry allows one to compute to a large extent all dynamical details encoded in the low energy effective action of a given formulation of the theory and to

${ }^{1}$ Work supported in part by EEC under TMR contract ERBFMRX-CT96-0045 (LNF Frascati, Politecnico di Torino and Univ. Genova) and by DOE grant DE-FGO3-91ER40662.

${ }^{2}$ Based on lectures given by S. Ferrara at the 5th Winter School on Mathematical Physics held at the Asia Pacific Center for Theoretical Physics, Seul (Korea), February 1997 
study the moduli (coupling constants) dependence of the BPS states. This latter property is important in order to study more dynamical questions such as phase transitions in the moduli space [4], [5], [6], [7], [8], [9] or properties of solitonic solutions of cosmological interest, such as extreme black holes [10] and their entropy. A major mathematical tool in these studies is the structure of supergravity theories in diverse dimensions [11] and with different numbers of supersymmetries. These theories have a central extension that gives an apparent violation of the Haag-Lopuszanski-Shonius theorem [12], since they include "central charges" that are not Lorentz-invariant 13. However, these charges are important because they are related to $p$-extended objects (for charges with $p$ antisymmetrized indices) whose dynamics is now believed to be as fundamental as that of points and strings 11. In fact point-like and string-like BPS states can be obtained by wrapping $p$ (or $p-1)$ of the dimensions of a $p$-extended object living in $D$ dimensions when $d \geq p$ dimensions have been compactified. It is the aim of this paper to give a detailed analysis of central extensions of different supergravities existing in arbitrary dimensions $4 \leq D<10$ in a unified framework and to study the moduli dependence of the BPS mass per unit of $p$-volume of generic BPS p-branes existing in a given theory. A basic tool in our investigation will be an exploitation of "duality symmetries" [14], [15] (rephrased nowadays as U-duality) of the underlying supergravity theory which, for a theory with more than 8 supercharges, takes the form of a discrete subgroup of the continuous isometries of the scalar field sigma model of the theory [16]. Duality symmetries, which rotate electric and magnetic charges, correspond, in a string context, to certain perturbative or nonperturbatives symmetries of the BPS spectrum, playing a crucial role in the study of string dynamics.

The basic focus of our approach is that the central extension of the supersymmetry algebra [12] 17] is encoded in the supergravity transformation rules. The latter can be derived from supersymmetric Bianchi identities, even if the complete lagrangian has not yet been derived. Of course a careful study of these identities also allows a complete determination of the lagrangian, whenever it exists. Among the novelties of this analysis is a new formulation of $D=4, N$-extended theories with $N>2$, in which a manifest symplectic formulation is used. In particular all these theories have in common a flat symplectic bundle which encodes the differential relations among the symplectic sections and therefore among the central and matter charges. In this respect the $N=2$ case, related to Special Geometry [18], [19], simply differs from the $N>2$ cases by the fact that the base space is not necessarily a coset space. This is related to the physical fact that $N=2$ Special Geometry suffers quantum corrections. For higher dimensional theories, relations between central and matter charges for different $p$-extended objects can be derived in in a way strictly analogous to that presented in this lecture in $D=4$ [20].

The application of this geometrical setting to black-hole physics, in particular to the determination of the black-hole entropy and fixed scalars, see [21] is described in the second part of these lectures.

\section{Extended supergravities and their relations with superstrings, M-theory and F-theory}

It is worth while to recall the various compactifications of superstrings in $4 \leq D<10$ as well as of M-theory and their relation to extended supergravities and their duality 
symmetries. In the string context the latter symmetries are usually called $\mathrm{S}, \mathrm{T}$ and U-dualities. S-duality means exchange of small with large coupling constant, i.e. strongweak coupling duality. T-duality indicates the exchange of small with large volume of compactification while U-duality refers to the exchange of NS with RR scalars. The major virtue of space-time supersymmetry is that it links toghether these dualities; often some of them are interchanged in comparing dual theories in the nonperturbative regime. In this lecture we will only consider compactifications on smooth manifolds since the analysis is otherwise more complicated (and richer) due to additional states concentrated at the singular points of the moduli space. The key ingredient to compare different theories in a given space-time dimension is Poincaré duality, which converts a theory with a $(p+2)$-form into one with a $(D-p-2)$-form (and inverse coupling constant). For example, at $D=9$ Poincaré duality relates 4 - and 5-forms, at $D=7$ [2] 3- and 4 -forms and at $D=52$ - and 3 -forms [25]. These relations are closely related to the fact that type IIA and type IIB are T-dual at $D=9$ [26], heterotic on $T_{3}$ is dual to M-theory on $K 3$ at $D=7$ [2] and heterotic on $K 3 \times S_{1}$ is dual to M-theory on $C Y_{3}$ at $D=5$ [27], [9]. Let us consider dualities by first comparing theories with maximal supersymmetry (32 supersymmetries). An example is the duality between M-theory on $S_{1}$ at large radius and type IIA in $D=10$ at strong coupling. For the sequel we will omit the regime where these theories should be compared. We will just identify their low-energy effective action including BPS states. Further compactifying type IIA on $S_{1}$, it becomes equivalent to IIB on $S_{1}$ with inverse radius. This is the T-duality alluded to before. It merely comes by Poincaré duality, exchanging the five form of one theory (IIB) with the 4-form of the other theory (IIA). The interrelation between M-theory and type IIA and type IIB theories at $D \leq 9$ explains most of the symmetries of all maximally extended supergravities. At $D=8$ we have a maximal theory with U-duality [16] group $S l(3, \mathbb{Z}) \times S l(2, \mathbb{Z})$. The $S l(3, \mathbb{Z})$ has a natural interpretation from an M-theory point of view, since the $D=8$ theory is M-theory on $T_{3}$. On the other hand, the additional $S l(2, \mathbb{Z})$ which acts on the 4 -form and its dual has a natural interpretation from the type IIB theory on $T_{2}$, in which the $\operatorname{Sl}(2, \mathbb{Z})$ is related to the complex structure of the 2-torus 28]. At $D=7$ the $S l(5, \mathbb{Z})$ U-duality has no obvious interpretation unless we move to an F-theory setting [28] This is also the case for $D=6,5,4$, where the U-duality groups are $O(5,5 ; \mathbb{Z}), E_{6,6}(\mathbb{Z})$ and $E_{7,7}(\mathbb{Z})$ respectively. However, they share the property that the related continuous group has, as maximal compact subgroup, the automorphism group of the supersymmetry algebra, i.e. $S p(4), S p(4) \times S p(4), U s p(8)$ and $S U(8)$ respectively for $D=7,6,5$ and 4 . The $\mathrm{U}$-duality group for any $D$ corresponds to the series of $E_{11-D}$ Lie algebras whose quotient with the above automorphism group of the supersymmetry algebra provides the local description of the scalar fields moduli space [29]. Recently, a novel way to unravel the structure of the U-duality groups in terms of solvable Lie algebras has been proposed in [30]. Moving to theories with lower (16) supersymmetries, we start to have dualities among heterotic, M-theory and type II theories on manifolds preserving 16 supersymmetries. For $D=7$, heterotic theory on $T_{3}$ is "dual" to M-theory on $K 3$ in the same sense that M-theory is "dual" to type IIA at $D=10$. Here the coset space $O(1,1) \times \frac{O(3,19)}{O(3) \times O(19)}$ identifies the dilaton and Narain lattice of the heterotic string with the classical moduli space of $K 3$, the dilaton in one theory being related to the volume of $K 3$ of the other theory [2]. The heterotic string on $T_{4}$ is dual to type IIA on $K 3$. Here the coset space $O(1,1) \times \frac{O(4,20)}{O(4) \times O(20)}$ identifies the Narain lattice with 
the "quantum" moduli space of $K 3$ (including torsion). The $O(1,1)$ factor again relates the dilaton to the $K 3$ volume. A similar situation occurs for the theories at $D=5$.

At $D=4$ a new phenomenon occurs since the classical moduli space $\frac{S U(1,1)}{U(1)} \times \frac{O(6,22)}{O(6) \times O(22)}$ interchanges S-duality of heterotic string with T-duality of type IIA theory and U-duality of type IIB theory [31]. If we compare theories with 8 supersymmetries, we may at most start with $D=6$. On the heterotic side this would correspond to $K 3$ compactification. However at $D=6$ no M-theory or type II correspondence is possible because we have no smooth manifolds of dimension 5 or 4 which reduce the original supersymmetry (32) by one quarter. The least we can do is to compare theories at $D=5$, where heterotic theory on $K 3 \times S_{1}$ can be compared [27] and in fact is dual, to M-theory on a CalabiYau threefold which is a $K 3$ fibration [32]. Finally, at $D=4$ the heterotic string on $K 3 \times T_{2}$ is dual to type IIA (or IIB) on a Calabi-Yau threefold (or its mirror) [33], [34], [35. It is worth noticing that these "dualities" predict new BPS states as well as they identify perturbative BPS states of one theory with non-perturbative ones in the dual theory. A more striking correspondence is possible if we further assume the existence of 12 dimensional $\mathrm{F}$-theory such that its compactification on $T_{2}$ gives type IIB at $D=10$ [3]. In this case we can relate the heterotic string on $T_{2}$ at $D=8$ to F-theory on $K 3$ and the heterotic string at $D=6$ on $K 3$ with F-theory on a Calabi-Yau threefold [8], [36]. To make these comparisons one has to further assume that the smooth manifolds of F-theory are elliptically fibered [8]. An even larger correspondence arises if we also include type I strings [37] and D-branes [38] in the game. However we will not further comment on the other correspondences relating all string theories with $\mathrm{M}$ and $\mathrm{F}$ theory.

\section{Duality symmetries and central charges in diverse dimensions}

\subsection{The general framework}

In this section we study the general group theoretical framework for the construction of the graviphotons and matter vectors appearing in the transformation laws of the fermion fields, the central and matter charges and the relations among them in any dimensions. After these preliminaries, we concentrate on the four dimensional theories, giving the details of the construction in this case. The details for the higher dimensional cases can be found in [20].

All supergravity theories contain scalar fields whose kinetic Lagrangian is described by $\sigma$-models of the form $G / H$, with the exception of $D=4, N=1,2$ and $D=5, N=2$. Here $G$ is a non compact group acting as an isometry group on the scalar manifold while $H$, the isotropy subgroup, is of the form:

$$
H=H_{\text {Aut }} \otimes H_{\text {matter }}
$$

$H_{\text {Aut }}$ being the automorphism group of the supersymmetry algebra while $H_{\text {matter }}$ is related to the matter multiplets. (Of course $H_{\text {matter }}=\mathbb{1}$ in all cases where supersymmetric matter doesn't exist, namely $N>4$ in $D=4,5$ and in general in all maximally extended supergravities). The coset manifolds $G / H$ and the automorphism groups for various supergravity theories for any $D$ and $N$ can be found in the literature (see for instance [11, 
[39]). As it is well known, the group $\mathrm{G}$ acts linearly on the $(n=p+2)$-forms field strengths $H_{a_{1} \cdots a_{n}}^{\Lambda}$ corresponding to the various $(p+1)$-forms appearing in the gravitational and matter multiplets. Here and in the following the index $\Lambda$ runs over the dimensions of some representation of the duality group $G$. The true duality symmetry (U-duality), acting on integral quantized electric and magnetics charges, is the restriction of the continuous group $G$ to the integers [16]. The moduli space of these theories is $G(\mathbb{Z}) \backslash G / H$.

All the properties of the given supergravity theories for fixed $D$ and $N$ are completely fixed in terms of the geometry of $G / H$, namely in terms of the coset representatives $L$ satisfying the relation:

$$
L\left(\phi^{\prime}\right)=g L(\phi) h(g, \phi)
$$

where $g \in G, h \in H$ and $\phi^{\prime}=\phi^{\prime}(\phi), \phi$ being the coordinates of $G / H$. Note that the scalar fields in $G / H$ can be assigned, in the linearized theory, to linear representations $R_{H}$ of the local isotropy group $H$ so that $\operatorname{dim} R_{H}=\operatorname{dim} G-\operatorname{dim} H$ (in the full theory, $R_{H}$ is the representation which the vielbein of $G / H$ belongs to).

As explained in the following, the kinetic metric for the $(p+2)$-forms $H^{\Lambda}$ is fixed in terms of $L$ and the physical field strengths of the interacting theories are "dressed" with scalar fields in terms of the coset representatives. This allows us to write down the central charges associated to the $(p+1)$-forms in the gravitational multiplet in a neat way in terms of the geometrical structure of the moduli space. In an analogous way also the matter $(p+1)$-forms of the matter multiplets give rise to charges which, as we will see, are closely related to the central charges. Note that when $p>1$ the central charges do not appear in the usual supersymmetry algebra, but in the extended version of it containing central generators $Z_{a_{1} \cdots a_{p}}$ associated to $p$-dimensional extended objects $\left(a_{1} \cdots a_{p}\right.$ are a set of space-time antisymmetric Lorentz indices) 440, 14, 13, 42, 43

Our main goal is to write down the explicit form of the dressed charges and to find relations among them analogous to those worked out in $D=4, N=2$ by means of the Special Geometry relations [44] 33].

To any $(p+2)$-form $H^{\Lambda}$ we may associate a magnetic charge $((D-p-4)$-brane $)$ and an electric ( $p$-brane) charge given respectively by:

$$
g^{\Lambda}=\int_{S^{p+2}} H^{\Lambda} \quad e_{\Lambda}=\int_{S^{D-p-2}} \mathcal{G}_{\Lambda}
$$

where $\mathcal{G}_{\Lambda}=\frac{\partial \mathcal{L}}{\partial H^{\Lambda}}$.

These charges however are not the physical charges of the interacting theory; the latter ones can be computed by looking at the transformation laws of the fermion fields, where the physical field-strengths appear dressed with the scalar fields [61, [20]. Let us first introduce the central charges: they are associated to the dressed $(p+2)$-forms $T_{A B}^{i}$ appearing in the supersymmetry transformation law of the gravitino 1-form. Quite generally we have, for any $D$ and $N$ :

$$
\delta \psi_{A}=D \epsilon_{A}+\sum_{i} c_{i} T_{A B \mid a_{1} \cdots a_{n_{i}}}^{i} \Delta^{a a_{1} \cdots a_{n_{i}}} \epsilon^{B} V_{a}+\cdots
$$

where:

$$
\Delta_{a a_{1} \cdots a_{n}}=\left(\Gamma_{a a_{1} \cdots a_{n}}-\frac{n}{n-1}(D-n-1) \delta_{\left[a_{1}\right.}^{a} \Gamma_{\left.a_{2} \cdots a_{n}\right]}\right) .
$$


Here $D$ is the covariant derivative in terms of the space-time spin connection and the composite connection of the automorphism group $H_{A u t}, c_{i}$ are coefficients fixed by supersymmetry, $V^{a}$ is the space-time vielbein, $A=1, \cdots, N$ is the index acted on by the automorphism group, $\Gamma_{a_{1} \cdots a_{n}}$ are $\gamma$-matrices in the appropriate dimensions, and the sum runs over all the $(p+2)$-forms appearing in the gravitational multiplet. Here and in the following the dots denote trilinear fermion terms. Each $n$-form field-strength $T_{A B}^{i}$ is constructed by dressing the bare field-strengths $H^{\Lambda}$ with the coset representative $L(\phi)$ of $G / H, \phi$ denoting a set of coordinates of $G / H$. In particular, for any $p$, except for $D / 2=p+2$, we have:

$$
T_{A B}^{i}=L_{A B \Lambda_{i}}(\phi) H^{\Lambda_{i}}
$$

where we have used the following decomposition of $L$ :

$$
L=\left(L_{A B}^{\Lambda}, L_{I}^{\Lambda}\right) \quad L^{-1}=\left(L_{A B \Lambda}, L_{I \Lambda}\right)
$$

Here $L_{\Sigma}^{\Lambda}$ belongs to the representation of $G$ under which the $(p+2)$-forms $H^{\Lambda}$ transform irreducibly and the couple of indices $A B$ and $I$ refer to the transformation properties of $L$ under the right action of $H_{A u t} \times H_{\text {matter }}$. More precisely, the couple of indices $A B$ transform in the twofold tensor representation of $H_{A u t}$, which in general is a $U s p(N)$ group (except in $D=8, N=1$ and $D=9, N=2$ theories where $H_{A u t}$ is $U(1)$ or $S U(2) \times U(1)$ respectively). and $I$ is an index in the fundamental representation of $H_{\text {matter }}$ which in general is an orthogonal group. Note that in absence of matter multiplets $L \equiv\left(L_{A B}^{\Lambda}\right)$. In all these cases $(D / 2 \neq p+2)$ the kinetic matrix of the $(p+2)$-forms $H^{\Lambda}$ is given in terms of the coset representatives as follows:

$$
\frac{1}{2} L_{A B \Lambda} L_{\Sigma}^{A B}-L_{I \Lambda} L_{\Sigma}^{I}=\mathcal{N}_{\Lambda \Sigma}
$$

with the indices of $H_{A u t}$ raised and lowered with the appropriate metric of $H_{A u t}$ in the given representation. For maximally extended supergravities $\mathcal{N}_{\Lambda \Sigma}=L_{A B \Lambda} L_{\Sigma}^{A B}$. Note that both for matter coupled and maximally extended supergravities we have:

$$
L_{\Lambda A B}=\mathcal{N}_{\Lambda \Sigma} L_{A B}^{\Sigma}
$$

When $G$ contains an orthogonal factor $O(m, n)$, what happens for matter coupled supergravities in $D=5,7,8,9$, where $G=O(10-D, n) \times O(1,1)$ and in all the matter coupled $D=6$ theories, the coset representatives of the orthogonal group satisfy:

$$
\begin{aligned}
L^{t} \eta L=\eta & \rightarrow L_{r \Lambda} L_{r \Sigma}-L_{I \Lambda} L_{I \Sigma}=\eta_{\Lambda \Sigma} \\
L^{t} L=\mathcal{N} & \rightarrow L_{r \Lambda} L_{r \Sigma}+L_{I \Lambda} L_{I \Sigma}=\mathcal{N}_{\Lambda \Sigma}
\end{aligned}
$$

where $\eta=\left(\begin{array}{cc}\mathbb{1}_{m \times m} & 0 \\ 0 & -\mathbb{1}_{n \times n}\end{array}\right)$ is the $O(m, n)$ invariant metric and $A=1, \cdots, m ; I=$ $1, \cdots, n$ (In particular, setting the matter to zero, we have in these cases $\mathcal{N}_{\Lambda \Sigma}=\eta_{\Lambda \Sigma}$ ). In these cases we have:

$$
L_{A B}^{\Lambda}=L_{r}^{\Lambda}\left(\gamma^{r}\right)_{A B},
$$

$\left(\gamma^{r}\right)_{A B}$ being the $\gamma$-matrices intertwining between orthogonal and $U S p(N)$ indices.

When $D$ is even and $D / 2=p+2$ the previous formulae in general require modifications, since in that case we have the complication that the action of $G$ on the $p+2=D / 2$-forms 
( $D$ even) is realized through the embedding of $G$ in $S p(2 n, \mathbb{R})(p$ even) or $O(n, n)$ ( $p$ odd) groups [45], 446].

This happens for $D=4, N>1, D=6, N=(2,2)$ and the maximally extended $D=6$ and $D=8$ supergravities. 3 The necessary modifications for the embedding are worked out in section 3.2 .

Coming back to the case $D / 2 \neq p+2$, it is now straightforward to compute the central charges.

Indeed, the magnetic central charges for BPS saturated $(D-p-4)$-branes can be now defined (modulo numerical factors to be fixed in each theory) by integration of the dressed field strengths as follows:

$$
Z_{(m) A B}^{(i)}=\int_{S^{p+2}} T_{A B}^{i}=\int_{S^{p+2}} L_{\Lambda_{i} A B}(\phi) H^{\Lambda_{i}}=L_{\Lambda_{i} A B}\left(\phi_{0}\right) g^{\Lambda_{i}}
$$

where $\phi_{0}$ denote the v.e.v. of the scalar fields, namely $\phi_{0}=\phi(\infty)$ in a given background. The corresponding electric central charges are:

$$
Z_{(e) A B}^{(i)}=\int_{S^{D-p-2}} L_{\Lambda_{i} A B}(\phi)^{\star} H^{\Lambda_{i}}=\int_{S^{D-p-2}} \mathcal{N}_{\Lambda_{i} \Sigma_{i}} L_{A B}^{\Lambda_{i}}(\phi){ }^{\star} H^{\Sigma_{i}}=L_{A B}^{\Lambda_{i}}\left(\phi_{0}\right) e_{\Lambda_{i}}
$$

These formulae make it explicit that $L_{A B}^{\Lambda}$ and $L_{\Lambda A B}$ are related by electric-magnetic duality via the kinetic matrix.

Note that the same field strengths $T_{A B}^{i}$ which appear in the gravitino transformation laws are also present in the dilatino transformation laws in the following way:

$$
\delta \chi_{A B C}=\cdots+\sum_{i} b_{i} L_{\Lambda_{i} A B}(\phi) H_{a_{1} \cdots a_{n_{i}}}^{\Lambda_{i}} \Gamma^{a_{1} \cdots a_{n_{i}}} \epsilon_{C}+\cdots
$$

In an analogous way, when vector multiplets are present, the matter vector field strengths are dressed with the columns $L_{\Lambda I}$ of the coset element (3.7) and they appear in the transformation laws of the gaugino fields:

$$
\delta \lambda_{A}^{I}=c_{1} \Gamma^{a} P_{A B, i}^{I} \partial_{a} \phi^{i} \epsilon^{B}+c_{2} L_{\Lambda}^{I}(\phi) F_{a b}^{\Lambda} \Gamma^{a b} \epsilon_{A}+\cdots
$$

where $P_{A B}^{I}=P_{A B, i}^{I} d \phi^{i}$ (see eq. (3.23) in the following) is the vielbein of the coset manifold spanned by the scalar fields of the vector multiplets, $F_{a b}^{\Lambda}$ is the field--strength of the matter photons and $c_{1}, c_{2}$ are constants fixed by supersymmetry (in $D=6, N=(2,0)$ and $N=(4,0)$ the 2 -form $F_{a b}^{\Lambda} \Gamma^{a b}$ is replaced by the 3 -form $\left.H_{a b c}^{\Lambda} \Gamma^{a b c}\right)$. In the same way as for central charges, one finds the magnetic matter charges:

$$
Z_{(m) A}^{I}=\int_{S^{p+2}} L_{\Lambda}^{I} F^{\Lambda}=L_{\Lambda}^{I}\left(\phi_{0}\right) g^{\Lambda}
$$

while the electric matter charges are:

$$
Z_{(e) I}=\int_{S^{D-p-2}} L_{\Lambda I}(\phi)^{\star} F^{\Lambda}=\int_{S^{D-p-2}} \mathcal{N}_{\Lambda \Sigma} L_{I}^{\Lambda}(\phi){ }^{\star} F^{\Sigma}=L_{I}^{\Lambda}\left(\phi_{0}\right) e_{\Lambda}
$$

\footnotetext{
${ }^{3}$ The 6 dimensional theories $N=(2,0)$ and $N=(4,0)$ do not require such embedding since the 3-forms $H^{\Lambda}$ have definite self-duality and no lagrangian exists. For these theories the formulae of the previous odd dimensional cases are valid.
} 
The important fact to note is that the central charges and matter charges satisfy relations and sum rules analogous to those derived in $D=4, N=2$ using Special Geometry techniques which we review in the following [44]. They are inherited from the properties of the coset manifolds $G / H$, namely from the differential and algebraic properties satisfied by the coset representatives $L_{\Sigma}^{\Lambda}$. Indeed, for a general coset manifold we may introduce the left-invariant 1-form $\Omega=L^{-1} d L$ satisfying the relation (see for instance [39]):

$$
d \Omega+\Omega \wedge \Omega=0
$$

where

$$
\Omega=\omega^{i} T_{i}+P^{\alpha} T_{\alpha}
$$

$T_{i}, T_{\alpha}$ being the generators of $G$ belonging respectively to the Lie subalgebra $\mathbb{H}$ and to the coset space algebra $\mathbb{K}$ in the Cartan decomposition

$$
\mathbb{G}=\mathbb{H}+\mathbb{K}
$$

$\mathbb{G}$ being the Lie algebra of $G$. Here $\omega^{i}$ is the $\mathbb{H}$ connection and $P^{\alpha}$, in the representation $R_{H}$ of $H$, is the vielbein of $G / H$. Since in all the cases we will consider $G / H$ is a symmetric space $([\mathbb{K}, \mathbb{K}] \subset \mathbb{H}), \omega^{i} C_{i}{ }^{\alpha \beta}\left(C_{i}{ }^{\alpha \beta}\right.$ being the structure constants of $\left.G\right)$ can be identified with the Riemannian spin connection of $G / H$.

Suppose now we have a matter coupled theory. Then, using the decomposition (3.21), from $(3.19)$ and $(3.20)$ we get:

$$
d L_{A B}^{\Lambda}=\frac{1}{2} L_{C D}^{\Lambda} \omega_{A B}^{C D}+L_{I}^{\Lambda} P_{A B}^{I}
$$

where $P_{A B}^{I}$ is the vielbein on $G / H$ and $\omega^{C D}{ }_{A B}$ is the $\mathbb{H}$-connection in the given representation. It follows:

$$
\nabla^{(H)} L_{A B}^{\Lambda}=L_{I}^{\Lambda} P_{A B}^{I}
$$

where the derivative is covariant with respect to the $\mathbb{H}$-connection $\omega_{A B}^{C D}$. Using the definition of the magnetic dressed charges given in (3.13) we obtain:

$$
\nabla^{(H)} Z_{A B}=Z_{I} P_{A B}^{I}
$$

This is a prototype of the formulae one can derive in the various cases for matter coupled supergravities [20]. To illustrate one possible application of this kind of formulae let us suppose that in a given background preserving some number of supersymmetries $Z_{I}=0$ as a consequence of $\delta \lambda_{A}^{I}=0$. Then we find:

$$
\nabla^{(H)} Z_{A B}=0 \rightarrow d\left(Z_{A B} \bar{Z}^{A B}\right)=0
$$

that is the square of the central charge reaches an extremum with respect to the v.e.v. of the moduli fields. Backgrounds with such fixed scalars describe the horizon geometry of extremal black holes and behave as attractor points for the scalar fields evolution in the black hole geometry [22], [23], 224].

For the maximally extended supergravities there are no matter field-strengths and the previous differential relations become differential relations among central charges only. 
As an example, let us consider $D=5, N=8$ theory. In this case the Maurer-Cartan equations become:

$$
d L_{A B}^{\Lambda}=\frac{1}{2} L_{C D}^{\Lambda} \Omega_{A B}^{C D}+\frac{1}{2} \bar{L}^{\Lambda C D} P_{C D A B}
$$

where the coset representative is taken in the $27 \times 27$ fundamental representation of $E_{6}$, $\Omega_{A B}^{C D}=2 Q_{[c}^{[A} \delta_{D]}^{B]}, A B$ is a couple of antisymmetric symplectic-traceless $U S p(8)$ indices, $Q_{B}^{A}$ is the $U S p(8)$ connection and the vielbein $P_{C D A B}$ is antisymmetric, $\mathbb{C}_{A B}$-traceless and pseudo-real. Note that $\left(L_{C D}^{\Lambda}\right)^{*}=L^{\Lambda C D}$. Therefore we get:

$$
\nabla^{(H)} L_{A B}^{\Lambda}=\frac{1}{2} \bar{L}^{\Lambda C D} P_{C D A B}
$$

that is:

$$
\nabla^{(H)} Z_{A B}=\frac{1}{2} \bar{Z}^{C D} P_{C D A B}
$$

This relation implies that the vanishing of a subset of central charges forces the vanishing of the covariant derivatives of some other subset. Typically, this happens in some supersymmetry preserving backgrounds where the requirement $\delta \chi_{A B C}=0$ corresponds to the vanishing of just a subset of central charges. Finally, from the coset representatives relations (3.8) (3.10) it is immediate to obtain sum rules for the central and matter charges which are the counterpart of those found in $N=2, D=4$ case using Special Geometry 444. Indeed, let us suppose e.g. that the group $G$ is $G=O(10-D, n) \times O(1,1)$, as it happens in general for all the minimally extended supergravities in $7 \leq D \leq 9, D=6$ type $I I A$ and $D=5, N=2$. The coset representative is now a tensor product $L \rightarrow e^{\sigma} L$, where $e^{\sigma}$ parametrizes the $O(1,1)$ factor.

We have, from (3.10)

$$
L^{t} \eta L=\eta
$$

where $\eta$ is the invariant metric of $O(10-D, n)$ and from (3.8)

$$
e^{-2 \sigma}\left(L^{t} L\right)_{\Lambda \Sigma}=\mathcal{N}_{\Lambda \Sigma}
$$

Using the decomposition (3.21) one finds:

$$
\begin{gathered}
\frac{1}{2} Z_{A B} Z_{A B}-Z_{I} Z_{I}=g^{\Lambda} \eta_{\Lambda \Sigma} g^{\Sigma} e^{-2 \sigma} \\
\frac{1}{2} Z_{A B} Z_{A B}+Z_{I} Z_{I}=g^{\Lambda} \mathcal{N}_{\Lambda \Sigma} g^{\Sigma}
\end{gathered}
$$

In more general cases analogous relations of the same kind can be derived 20.

\subsection{The embedding procedure in $D=4$}

In this subsection we work out the modifications to the formalism developed in the previous subsection in the case $D / 2=p+2$, which derive from the embedding procedure 45 of the group $G$ in $S p(2 n, \mathbb{R})$ ( $p$ even) or in $O(n, n)$ ( $p$ odd). We concentrate on $D=4$, while for $D=6,8$ we refer to ref. [20]. Furthermore we show that the flat symplectic bundle formalism of the $D=4, N=2$ Special Geometry case [18], [19] can be extended to $N>2$ theories. The $N=2$ case differs from the other higher $N$ extensions by the fact that the base space of the flat symplectic bundle is not in general a coset manifold. 
Let us analyze the structure of the four dimensional theories.

In $D=4, N>2$ we may decompose the vector field-strengths in self-dual and anti self-dual parts:

$$
F^{\mp}=\frac{1}{2}\left(F \mp \mathrm{i}^{\star} F\right)
$$

According to the Gaillard-Zumino construction, $G$ acts on the vector $\left(F^{-\Lambda}, \mathcal{G}_{\Lambda}^{-}\right.$) (or its complex conjugate) as a subgroup of $\operatorname{Sp}\left(2 n_{v}, \mathbb{R}\right)\left(n_{v}\right.$ is the number of vector fields) with duality transformations interchanging electric and magnetic field-strengths:

$$
\mathcal{S}\left(\begin{array}{c}
F^{-\Lambda} \\
\mathcal{G}_{\Lambda}^{-}
\end{array}\right)=\left(\begin{array}{c}
F^{-\Lambda} \\
\mathcal{G}_{\Lambda}^{-}
\end{array}\right)^{\prime}
$$

where:

$$
\begin{gathered}
\mathcal{G}_{\Lambda}^{-}=\overline{\mathcal{N}}_{\Lambda \Sigma} F^{-\Sigma} \\
\mathcal{G}_{\Lambda}^{+}=\mathcal{N}_{\Lambda \Sigma} F^{+\Sigma} \\
\mathcal{S}=\left(\begin{array}{cc}
A & B \\
C & D
\end{array}\right) \in G \subset S p\left(2 n_{v}, \mathbb{R}\right) \rightarrow\left\{\begin{array}{l}
A^{t} C-C^{t} A=0 \\
B^{t} D-D^{t} B=0 \\
A^{t} D-C^{t} B=1
\end{array}\right.
\end{gathered}
$$

and $\mathcal{N}_{\Lambda \Sigma}$, is the symmetric matrix appearing in the kinetic part of the vector Lagrangian:

$$
\mathcal{L}_{\text {kin }}=\mathrm{i} \overline{\mathcal{N}}_{\Lambda \Sigma} F^{-\Lambda} F^{-\Sigma}+\text { h.c. }
$$

If $L(\phi)$ is the coset representative of $G$ in some representation, $S$ represents the embedded coset representative belonging to $S p\left(2 n_{v}, \mathbb{R}\right)$ and in each theory, $A, B, C, D$ can be constructed in terms of $L(\phi)$. Using a complex basis in the vector space of $\operatorname{Sp}\left(2 n_{v}\right)$, we may rewrite the symplectic matrix as an $U \operatorname{sp}\left(n_{v}, n_{v}\right)$ element:

$$
U=\frac{1}{\sqrt{2}}\left(\begin{array}{ll}
f+\mathrm{i} h & \bar{f}+\mathrm{i} \bar{h} \\
f-\mathrm{i} h & \bar{f}-\mathrm{i} \bar{h}
\end{array}\right)=\mathcal{A}^{-1} S \mathcal{A}
$$

where:

$$
\begin{aligned}
f & =\frac{1}{\sqrt{2}}(A-\mathrm{i} B) \\
h & =\frac{1}{\sqrt{2}}(C-\mathrm{i} D) \\
\mathcal{A} & =\left(\begin{array}{cc}
1 & 1 \\
-\mathrm{i} & \mathrm{i}
\end{array}\right)
\end{aligned}
$$

The requirement $U \in U \operatorname{sp}\left(n_{v}, n_{v}\right)$ implies:

$$
\left\{\begin{aligned}
i\left(f^{\dagger} h-h^{\dagger} f\right) & =\mathbb{1} \\
\left(f^{\dagger} \bar{h}-h^{\dagger} \bar{f}\right) & =0
\end{aligned}\right.
$$

The $n_{v} \times n_{v}$ subblocks of $\mathrm{U}$ are submatrices $f, h$ which can be decomposed with respect to the isotropy group $H_{\text {Aut }} \times H_{\text {matter }}$ in the same way as $L$ in equation (3.7), namely:

$$
\begin{aligned}
f & =\left(f_{A B}^{\Lambda}, f_{I}^{\Lambda}\right) \\
h & =\left(h_{\Lambda A B}, h_{\Lambda I}\right)
\end{aligned}
$$


where $A B$ are indices in the antisymmetric representation of $H_{A u t}=S U(N) \times U(1)$ and $I$ is an index of the fundamental representation of $H_{\text {matter }}$. Upper $S U(N)$ indices label objects in the complex conjugate representation of $S U(N):\left(f_{A B}^{\Lambda}\right)^{*}=f^{\Lambda A B}$ etc.

Note that we can consider $\left(f_{A B}^{\Lambda}, h_{\Lambda A B}\right)$ and $\left(f_{I}^{\Lambda}, h_{\Lambda I}\right)$ as symplectic sections of a $S p\left(2 n_{v}, \mathbb{R}\right)$ bundle over $G / H$. We will see in the following that this bundle is actually flat. The real embedding given by $S$ is appropriate for duality transformations of $F^{ \pm}$ and their duals $\mathcal{G}^{ \pm}$, according to equations (3.36), (3.35), while the complex embedding in the matrix $U$ is appropriate in writing down the fermion transformation laws and supercovariant field--strengths. The kinetic matrix $\mathcal{N}$, according to Gaillard-Zumino 45, turns out to be:

$$
\mathcal{N}=h f^{-1}, \quad \mathcal{N}=\mathcal{N}^{t}
$$

and transforms projectively under $S p\left(2 n_{v}, \mathbb{R}\right)$ duality rotations:

$$
\mathcal{N}^{\prime}=(C+D \mathcal{N})(A+B \mathcal{N})^{-1}
$$

By using (3.40)and (3.42) we find that

$$
\left(f^{t}\right)^{-1}=\mathrm{i}(\mathcal{N}-\overline{\mathcal{N}}) \bar{f}
$$

which is the analogous of equation (3.9), that is

$$
\begin{aligned}
f_{A B \Lambda} & \equiv\left(f^{-1}\right)_{A B \Lambda}=\mathrm{i}(\mathcal{N}-\overline{\mathcal{N}})_{\Lambda \Sigma} \bar{f}_{A B}^{\Sigma} \\
f_{I \Lambda} & \equiv\left(f^{-1}\right)_{I \Lambda}=\mathrm{i}(\mathcal{N}-\overline{\mathcal{N}})_{\Lambda \Sigma} \bar{f}_{I}^{\Sigma}
\end{aligned}
$$

It follows that the dressing factor $\left(L^{\Lambda}\right)^{-1}=\left(L_{\Lambda A B}, L_{\Lambda I}\right)$ in equation (3.4) which was given by the inverse coset representative in the defining representation of $G$ has to be replaced by the analogous inverse representative $\left(f_{\Lambda A B}, f_{\Lambda I}\right)$ when, as in the present $D=4$ case, we have to embed $G$ in $S p(2 n, \mathbb{R})$. As a consequence, in the transformation law of gravitino (3.4), dilatino (3.15) and gaugino (3.16) we perform the following replacement:

$$
\left(L_{\Lambda A B}, L_{\Lambda I}\right) \rightarrow\left(f_{\Lambda A B}, f_{\Lambda I}\right)
$$

In particular, the dressed graviphotons and matter self-dual field-strengths take the symplectic invariant form:

$$
\begin{aligned}
T_{A B}^{-} & =-\mathrm{i}\left(\bar{f}^{-1}\right)_{A B \Lambda} F^{-\Lambda}=f_{A B}^{\Lambda}(\mathcal{N}-\overline{\mathcal{N}})_{\Lambda \Sigma} F^{-\Sigma}=h_{\Lambda A B} F^{-\Lambda}-f_{A B}^{\Lambda} \mathcal{G}_{\Lambda}^{-} \\
T_{I}^{-} & =-\mathrm{i}\left(\bar{f}^{-1}\right)_{I \Lambda} F^{-\Lambda}=f_{I}^{\Lambda}(\mathcal{N}-\overline{\mathcal{N}})_{\Lambda \Sigma} F^{-\Sigma}=h_{\Lambda I} F^{-\Lambda}-f_{I}^{\Lambda} \mathcal{G}_{\Lambda}^{-} \\
\bar{T}_{A B}^{+} & =\left(T_{A B}^{-}\right)^{*} \\
\bar{T}_{I}^{+} & =\left(T_{I}^{-}\right)^{*}
\end{aligned}
$$

(Obviously, for $N>4, L_{\Lambda I}=f_{\Lambda I}=T_{I}=0$ ). To construct the dressed charges one integrates $T_{A B}=T_{A B}^{+}+T_{A B}^{-}$and (for $N=3,4$ ) $T_{I}=T_{I}^{+}+T_{I}^{-}$on a large 2-sphere. For this purpose we note that

$$
\begin{aligned}
T_{A B}^{+} & =h_{\Lambda A B} F^{+\Lambda}-f_{A B}^{\Lambda} \mathcal{G}_{\Lambda}^{+}=0 \\
T_{I}^{+} & =h_{\Lambda I} F^{+\Lambda}-f_{I}^{\Lambda} \mathcal{G}_{\Lambda}^{+}=0
\end{aligned}
$$


as a consequence of eqs. (3.42), (3.35). Therefore we have:

$$
\begin{aligned}
Z_{A B} & =\int_{S^{2}} T_{A B}=\int_{S^{2}}\left(T_{A B}^{+}+T_{A B}^{-}\right)=\int_{S^{2}} T_{A B}^{-}=h_{\Lambda A B} g^{\Lambda}-f_{A B}^{\Lambda} e_{\Lambda} \\
Z_{I} & =\int_{S^{2}} T_{I}=\int_{S^{2}}\left(T_{I}^{+}+T_{I}^{-}\right)=\int_{S^{2}} T_{I}^{-}=h_{\Lambda I} g^{\Lambda}-f_{I}^{\Lambda} e_{\Lambda} \quad(N \leq 4)
\end{aligned}
$$

where:

$$
e_{\Lambda}=\int_{S^{2}} \mathcal{G}_{\Lambda}, \quad g^{\Lambda}=\int_{S^{2}} F^{\Lambda}
$$

and the sections $\left(f^{\Lambda}, h_{\Lambda}\right)$ on the right hand side now depend on the v.e.v.'s of the scalar fields $\phi^{i}$. We see that the central and matter charges are given in this case by symplectic invariants and that the presence of dyons in $D=4$ is related to the symplectic embedding. In the case $D / 2 \neq p+2, D$ even, or $D$ odd, we were able to derive the differential relations (3.24), (3.28) among the central and matter charges using the Maurer-Cartan equations (3.23), (3.27). The same can be done in the present case using the embedded coset representative $U$. Indeed, let $\Gamma=U^{-1} d U$ be the $U s p\left(n_{v}, n_{v}\right)$ Lie algebra left invariant one form satisfying:

$$
d \Gamma+\Gamma \wedge \Gamma=0
$$

In terms of $(f, h) \Gamma$ has the following form:

$$
\Gamma \equiv U^{-1} d U=\left(\begin{array}{cc}
\mathrm{i}\left(f^{\dagger} d h-h^{\dagger} d f\right) & \mathrm{i}\left(f^{\dagger} d \bar{h}-h^{\dagger} d \bar{f}\right) \\
-\mathrm{i}\left(f^{t} d h-h^{t} d f\right) & -\mathrm{i}\left(f^{t} d \bar{h}-h^{t} d \bar{f}\right)
\end{array}\right) \equiv\left(\begin{array}{cc}
\Omega^{(H)} & \overline{\mathcal{P}} \\
\mathcal{P} & \bar{\Omega}^{(H)}
\end{array}\right)
$$

where the $n_{v} \times n_{v}$ subblocks $\Omega^{(H)}$ and $\mathcal{P}$ embed the $H$ connection and the vielbein of $G / H$ respectively. This identification follows from the Cartan decomposition of the $U \operatorname{sp}\left(n_{v}, n_{v}\right)$ Lie algebra. Explicitly, if we define the $H_{A u t} \times H_{\text {matter }}$-covariant derivative of a vector $V=\left(V_{A B}, V_{I}\right)$ as:

$$
\nabla V=d V-V \omega, \quad \omega=\left(\begin{array}{cc}
\omega^{A B} & 0 \\
0 & \omega^{I}
\end{array}\right)
$$

we have:

$$
\Omega^{(H)}=\mathrm{i}\left[f^{\dagger}(\nabla h+h \omega)-h^{\dagger}(\nabla f+f \omega)\right]=\omega
$$

where we have used:

$$
\nabla h=\overline{\mathcal{N}} \nabla f ; \quad h=\mathcal{N} f
$$

and the fundamental identity (3.40). Furthermore, using the same relations, the embedded vielbein $\mathcal{P}$ can be written as follows:

$$
\mathcal{P}=-\mathrm{i}\left(f^{t} \nabla h-h^{t} \nabla f\right)=\mathrm{i} f^{t}(\mathcal{N}-\overline{\mathcal{N}}) \nabla f
$$

From (3.38) and (3.55), we obtain the $\left(n_{v} \times n_{v}\right)$ matrix equation:

$$
\begin{aligned}
\nabla(\omega)(f+\mathrm{i} h) & =(\bar{f}+\mathrm{i} \bar{h}) \mathcal{P} \\
\nabla(\omega)(f-\mathrm{i} h) & =(\bar{f}-\mathrm{i} \bar{h}) \mathcal{P}
\end{aligned}
$$

together with their complex conjugates. Using further the definition (3.41) we have:

$$
\begin{aligned}
\nabla(\omega) f_{A B}^{\Lambda} & =\bar{f}_{I}^{\Lambda} P_{A B}^{I}+\frac{1}{2} \bar{f}^{\Lambda C D} P_{A B C D} \\
\nabla(\omega) f_{I}^{\Lambda} & =\frac{1}{2} \bar{f}^{\Lambda A B} P_{A B I}+\bar{f}_{J}^{\Lambda} P_{I}^{J}
\end{aligned}
$$


where we have decomposed the embedded vielbein $\mathcal{P}$ as follows:

$$
\mathcal{P}=\left(\begin{array}{cc}
P_{A B C D} & P_{A B J} \\
P_{I A B} & P_{I J}
\end{array}\right)
$$

the subblocks being related to the vielbein of $G / H, P=L^{-1} \nabla^{(H)} L$, written in terms of the indices of $H_{A u t} \times H_{\text {matter }}$. Note that, since $f$ belongs to the unitary matrix $U$, we have: $\left(\bar{f}_{A B}^{\Lambda}, \bar{f}_{I}^{\Lambda}\right)=\left(f^{\Lambda A B}, f^{\Lambda I}\right)$. Obviously, the same differential relations that we wrote for $f$ hold true for the dual matrix $h$ as well.

Using the definition of the charges (3.51), (3.52) we then get the following differential relations among charges:

$$
\begin{aligned}
\nabla(\omega) Z_{A B} & =\bar{Z}_{I} P_{A B}^{I}+\frac{1}{2} \bar{Z}^{C D} P_{A B C D} \\
\nabla(\omega) Z_{I} & =\frac{1}{2} \bar{Z}^{A B} P_{A B I}+\bar{Z}_{J} P_{I}^{J}
\end{aligned}
$$

Depending on the coset manifold, some of the subblocks of (3.62) can be actually zero. For example in $N=3$ the vielbein of $G / H=\frac{S U(3, n)}{S U(3) \times S U(n) \times U(1)}$ 477 is $P_{I A B}(A B$ antisymmetric), $I=1, \cdots, n ; A, B=1,2,3$ and it turns out that $P_{A B C D}=P_{I J}=0$.

In $N=4, G / H=\frac{S U(1,1)}{U(1)} \times \frac{O(6, n)}{O(6) \times O(n)}$ 48], and we have $P_{A B C D}=\epsilon_{A B C D} P, P_{I J}=\bar{P} \delta_{I J}$, where $P$ is the Kählerian vielbein of $\frac{S U(1,1)}{U(1)},(A, \cdots, D S U(4)$ indices and $I, J O(n)$ indices $)$ and $P_{I A B}$ is the vielbein of $\frac{O(6, n)}{O(6) \times O(n)}$.

For $N>4$ (no matter indices) we have that $\mathcal{P}$ coincides with the vielbein $P_{A B C D}$ of the relevant $G / H$.

For the purpose of comparison of the previous formalism with the $N=2$ Special Geometry case, it is interesting to note that, if the connection $\Omega^{(H)}$ and the vielbein $\mathcal{P}$ are regarded as data of $G / H$, then the Maurer-Cartan equations (3.61) can be interpreted as an integrable system of differential equations for a section $V=\left(V_{A B}, V_{I}, \bar{V}^{A B}, \bar{V}^{I}\right)$ of the symplectic fiber bundle constructed over $G / H$. Namely the integrable system:

$$
\nabla\left(\begin{array}{c}
V_{A B} \\
V_{I B} \\
\bar{V}^{A B} \\
\bar{V}^{I}
\end{array}\right)=\left(\begin{array}{cccc}
0 & 0 & \frac{1}{2} P_{A B C D} & P_{A B J} \\
0 & 0 & \frac{1}{2} P_{I C D} & P_{I J} \\
\frac{1}{2} P^{A B C D} & P^{A B J} & 0 & 0 \\
\frac{1}{2} P^{I C D} & P^{I J} & 0 & 0
\end{array}\right)\left(\begin{array}{c}
V_{C D} \\
V_{J} \\
\bar{V}^{C D} \\
\bar{V}^{J}
\end{array}\right)
$$

has $2 n$ solutions given by $V=\left(f_{A B}^{\Lambda}, f_{I}^{\Lambda}\right),\left(h_{\Lambda A B}, h_{\Lambda_{I}}\right), \Lambda=1, \cdots, n$. The integrability condition (3.54) means that $\Gamma$ is a flat connection of the symplectic bundle. In terms of the geometry of $G / H$ this in turn implies that the $\mathbb{H}$-curvature, and hence the Riemannian curvature, is constant, being proportional to the wedge product of two vielbein.

Besides the differential relations (3.63), the charges also satisfy sum rules quite analogous to those found in [44 for the $N=2$ Special Geometry case.

The sum rule has the following form:

$$
\frac{1}{2} Z_{A B} \bar{Z}^{A B}+Z_{I} \bar{Z}^{I}=-\frac{1}{2} P^{t} \mathcal{M}(\mathcal{N}) P
$$

where $\mathcal{M}(\mathcal{N})$ and $P$ are:

$$
\mathcal{M}=\left(\begin{array}{cc}
\mathbb{1} & 0 \\
-\operatorname{Re} \mathcal{N} & \mathbb{1}
\end{array}\right)\left(\begin{array}{cc}
\operatorname{Im} \mathcal{N} & 0 \\
0 & \operatorname{Im} \mathcal{N}^{-1}
\end{array}\right)\left(\begin{array}{cc}
\mathbb{1} & -\operatorname{Re} \mathcal{N} \\
0 & \mathbb{1}
\end{array}\right)
$$




$$
P=\left(\begin{array}{l}
g^{\Lambda} \\
e_{\Lambda}
\end{array}\right)
$$

In order to obtain this result we just need to observe that from the fundamental identities (3.76) and from the definition of the kinetic matrix given in (3.105) it follows:

$$
\begin{aligned}
f f^{\dagger} & =\mathrm{i}(\mathcal{N}-\overline{\mathcal{N}})^{-1} \\
h h^{\dagger} & =\mathrm{i}\left(\overline{\mathcal{N}}^{-1}-\mathcal{N}^{-1}\right)^{-1} \equiv \mathrm{i} \mathcal{N}(\mathcal{N}-\overline{\mathcal{N}})^{-1} \overline{\mathcal{N}} \\
h f^{\dagger} & =\mathcal{N} f f^{\dagger} \\
f h^{\dagger} & =f f^{\dagger} \overline{\mathcal{N}}
\end{aligned}
$$

We note that the matrix $\mathcal{M}$ is a symplectic tensor and in this sense it is quite analogous to the matrix $\mathcal{N}_{\Lambda \Sigma}$ of the odd dimensional cases defined in equation (3.8). Indeed, one sees that the matrix $\mathcal{M}$ can be written as the symplectic analogous of $(3.8)$ :

$$
\mathcal{M}(\mathcal{N})=\left(\begin{array}{cc}
0 & \mathbb{1} \\
-\mathbb{1} & 0
\end{array}\right)\left(\begin{array}{l}
f \\
h
\end{array}\right)\left(\begin{array}{ll}
f & h
\end{array}\right)^{\dagger}\left(\begin{array}{cc}
0 & \mathbb{1} \\
-\mathbb{1} & 0
\end{array}\right)
$$

where $\left(\begin{array}{cc}0 & \mathbb{1} \\ -\mathbb{1} & 0\end{array}\right)\left(\begin{array}{l}f \\ h\end{array}\right)$ is the embedded object corresponding to the $L$ of equation (3.8)

The formalism we have developed so far for the $D=4, N>2$ theories is completely determined by the embedding of the coset representative of $G / H$ in $S p(2 n, \mathbb{R})$ and by the $U s p(n, n)$ embedded Maurer-Cartan equations (3.61). We want now to show that this formalism, and in particular the identities (3.40), the differential relations among charges (3.63) and the sum rules (3.65), are completely analogous to the Special Geometry relations of $N=2$ matter coupled supergravity [49], [18]. This follows essentially from the fact that, though that the scalar manifold $\mathcal{M}_{N=2}$ of the $N=2$ theory is not in general a coset manifold, nevertheless it has a symplectic structure identical to the $N>2$ theories. Furthermore we will show that the analogous of the Maurer-Cartan equations of $N>2$ theories are given in the $N=2$ case by the Picard-Fuchs equations [19 for the symplectic sections which enter in the definition of the Special Geometry flat symplectic bundle.

Indeed, let us recall that Special Geometry can be defined in terms of the holomorphic flat vector bundle of rank $2 n$ with structure group $S p(2 n, \mathbb{R})$ over a Kähler-Hodge manifold [18].

If we introduce the Special Geometry symplectic and covariantly holomorphic section:

$$
V \equiv\left(f^{\Lambda}, h_{\Lambda}\right)=\left(f^{\Lambda}\left(z^{i}, z^{\bar{\imath}}\right), h_{\Lambda}\left(z^{i}, z^{\bar{\imath}}\right)\right) ; \nabla_{\bar{\imath}} V=0 \quad \Lambda=1, \ldots, n
$$

and its covariant derivative with respect to the Kähler connection:

$$
\nabla_{i} V=\left(\partial_{i}+\frac{1}{2} \partial_{i} K\right) V \equiv\left(f_{i}^{\Lambda}, h_{\Lambda i}\right) \quad i=1, \cdots, n-1
$$

then, defining the $n \times n$ matrices:

$$
f_{\Sigma}^{\Lambda} \equiv\left(f^{\Lambda}, f_{\bar{\imath}}^{\Lambda}\right) ; \quad h_{\Lambda \Sigma} \equiv\left(h_{\Lambda}, h_{\Lambda \bar{\imath}}\right)
$$

the set of algebraic relations of Special Geometry can be written in matrix form as:

$$
\left\{\begin{aligned}
\mathrm{i}\left(f^{\dagger} h-h^{\dagger} f\right) & =\mathbb{1} \\
\left(f^{\dagger} \bar{h}-h^{\dagger} \bar{f}\right) & =0
\end{aligned}\right.
$$


Recalling equations (3.40) we see that the previous relations imply that the matrix $U$ :

$$
U=\frac{1}{\sqrt{2}}\left(\begin{array}{ll}
f+\mathrm{i} h & \bar{f}+\mathrm{i} \bar{h} \\
f-\mathrm{i} h & \bar{f}-\mathrm{i} \bar{h}
\end{array}\right)
$$

belongs to $U s p(n, n)$. In fact if we set $f^{\Lambda} \rightarrow f^{\Lambda} \epsilon_{A B} \equiv f_{A B}^{\Lambda}$ and flatten the indices of $\left(\bar{f}_{i}, f_{\bar{\imath}}\right)\left(\right.$ or $\left.\left(\bar{h}_{i}, h_{\bar{\imath}}\right)\right)$ with the Kählerian vielbein $P_{i}^{I}, \bar{P}_{\bar{\imath}}^{\bar{I}}$ :

$$
\left(\bar{f}_{I}^{\Lambda}, f_{\bar{I}}^{\Lambda}\right)=\left(\bar{f}_{i}^{\Lambda} P_{I}^{i}, f_{\bar{\imath}}^{\Lambda} \bar{P}_{\bar{I}}^{\bar{\imath}}\right), \quad P_{i}^{I} \bar{P}_{\bar{\jmath}}^{\bar{J}} \eta_{I \bar{J}}=g_{i \bar{\jmath}}
$$

where $\eta_{I \bar{J}}$ is the flat Kählerian metric and $P_{I}^{i}=\left(P^{-1}\right)_{i}^{I}$, the relations (3.76) are just a particular case of equations (3.40) since, for $N=2, H_{A u t}=S U(2) \times U(1)$, so that $f_{A B}^{\Lambda}$ is actually an $S U(2)$ singlet.

Let us now consider the analogous of the embedded Maurer-Cartan equations of $G / H$. Defining as before the matrix one-form $\Gamma=U^{-1} d U$ valued in the $U s p(n, n)$ Lie algebra, we see that the relation $d \Gamma+\Gamma \wedge \Gamma=0$ again implies a flat connection for the symplectic bundle over the Kähler-Hodge manifold. However, this does not imply anymore that the base manifold is a coset or a constant curvature manifold. Indeed, let us introduce the covariant derivative of the symplectic section $\left(f^{\Lambda}, f \bar{I}^{\Lambda}, \bar{f}^{\Lambda}, \bar{f}_{I}^{\Lambda}\right)$ with respect to the $U(1)$ Kähler connection $\mathcal{Q}$ and the spin connection $\omega^{I J}$ of $\mathcal{M}_{N=2}$ :

$$
\begin{gathered}
\nabla\left(f^{\Lambda}, f_{\bar{I}}^{\Lambda}, \bar{f}^{\Lambda}, \bar{f}_{I}^{\Lambda}\right)= \\
d\left(f^{\Lambda}, f_{\bar{I}}^{\Lambda}, \bar{f}^{\Lambda}, \bar{f}_{I}^{\Lambda}\right)+\left(f^{\Lambda}, f_{\bar{J}}^{\Lambda}, \bar{f}^{\Lambda}, \bar{f}_{J}^{\Lambda}\right)\left(\begin{array}{cccc}
\mathrm{i} \mathcal{Q} & 0 & 0 & 0 \\
0 & -\mathrm{i} \mathcal{Q} \delta_{\frac{I}{J}}+\omega^{\bar{I}} & 0 & 0 \\
0 & 0 & -\mathrm{i} \mathcal{Q} & 0 \\
0 & 0 & 0 & \mathrm{i} \mathcal{Q} \delta_{J}^{I}+\omega_{J}^{I}
\end{array}\right)
\end{gathered}
$$

where:

$$
\mathcal{Q}=-\frac{\mathrm{i}}{2}\left(\partial_{i} \mathcal{K} d z^{i}-\bar{\partial}_{\bar{\imath}} \mathcal{K} d \bar{z}^{\bar{\imath}}\right) \rightarrow d \mathcal{Q}=\mathrm{i} g_{i \bar{\jmath}} d z^{i} \wedge d \bar{z}^{\bar{z}}
$$

$\left(\mathcal{K}\right.$ is the Kähler potential) the Kähler weight of $\left(f^{\Lambda}, \bar{f}_{I}^{\Lambda}\right)$ and $\left(\bar{f}^{\Lambda}, f_{\bar{I}}^{\Lambda}\right)$ being $p=1$ and $p=-1$ respectively. Using the same decomposition as in equation (3.55) and eq.s (3.56), (3.20) we have in the $N=2$ case:

$$
\begin{aligned}
\Gamma & =\left(\begin{array}{ll}
\Omega & \overline{\mathcal{P}} \\
\mathcal{P} & \bar{\Omega}
\end{array}\right), \\
\Omega & =\omega=\left(\begin{array}{cc}
\mathrm{i} p \mathcal{Q} & 0 \\
0 & \mathrm{i} p \mathcal{Q} \delta_{J}^{I}+\bar{\omega}_{J}^{I}
\end{array}\right)
\end{aligned}
$$

For the embedded vielbein $\mathcal{P}$ we obtain:

$$
\mathcal{P}=-\mathrm{i}\left(f^{t} \nabla h-h^{t} \nabla f\right)=\mathrm{i} f^{t}(\mathcal{N}-\overline{\mathcal{N}}) \nabla f=\left(\begin{array}{cc}
0 & \bar{P}_{I} \\
\bar{P}^{\bar{J}} & \bar{P}_{I}^{J}
\end{array}\right)
$$

where $\bar{P}^{\bar{I}}$ is the $(0,1)$-form Kählerian vielbein while $\bar{P}_{I}^{\bar{J}} \equiv\left(f^{t}(\mathcal{N}-\overline{\mathcal{N}}) \nabla f\right)_{I}^{\bar{J}}$ is a oneform which in general cannot be expressed in terms of the vielbein $P^{I}$ and therefore represents a new geometrical quantity on $\mathcal{M}_{N=2}$. Note that we get zero in the first entry of equation (3.82) by virtue of the fact that the identity (3.76) implies $f^{\Lambda}(\mathcal{N}-\overline{\mathcal{N}})_{\Lambda \Sigma} f_{i}^{\Sigma}=0$ 
and that $f^{\Lambda}$ is covariantly holomorphic. If $\Omega$ and $\mathcal{P}$ are considered as data on $\mathcal{M}_{N=2}$ then we may interpret $\Gamma=U^{-1} d U$ as an integrable system of differential equations, namely:

$$
\nabla\left(V, V_{\bar{I}}, \bar{V}, \bar{V}_{I}\right)=\left(V, V_{\bar{J}}, \bar{V}, \bar{V}_{J}\right)\left(\begin{array}{cccc}
0 & 0 & 0 & \bar{P}_{I} \\
0 & 0 & \bar{P}^{\bar{J}} & \bar{P}_{I}^{J} \\
0 & P_{\bar{I}} & 0 & 0 \\
P^{J} & P_{\bar{I}}^{J} & 0 & 0
\end{array}\right)
$$

where the flat Kähler indices $I, \bar{I}, \cdots$ are raised and lowered with the flat Kähler metric $\eta_{I \bar{J}}$. As it is well known, this integrable system describes the Picard-Fuchs equations for the periods $\left(V, V_{\bar{I}}, \bar{V}, V_{I}\right)$ of the Calabi-Yau threefold with solution given by the $2 n$ symplectic vectors $V \equiv\left(f^{\Lambda}, h_{\Lambda}\right)$. The integrability condition $d \Gamma+\Gamma \wedge \Gamma=0$ gives three constraints on the Kähler base manifold:

$$
\begin{aligned}
d(\mathrm{i} \mathcal{Q})+\bar{P}^{I} \wedge P_{I} & =0 \rightarrow \partial_{\bar{\jmath}} \partial_{i} \mathcal{K}=P_{I, i} \bar{P}_{\overline{, j}}^{I}=g_{i \bar{\jmath}} \\
(d \omega+\omega \wedge \omega)_{\bar{I}}^{\bar{J}} & =P_{\bar{I}} \wedge \bar{P}^{\bar{J}}-\mathrm{i} d \mathcal{Q} \delta_{\bar{I}}^{\bar{J}}-\bar{P}^{\bar{J}} \wedge P^{L}{ }_{\bar{I}} \\
\nabla P_{\bar{I}}^{J} & =0
\end{aligned}
$$

Equation (3.84) implies that $\mathcal{M}_{N=2}$ is a Kähler-Hodge manifold. Equation (3.85), written with holomorphic and antiholomorphic curved indices, gives:

$$
R_{\bar{\imath} j \bar{k} l}=g_{\bar{\imath} l} g_{j \bar{k}}+g_{\bar{k} l} g_{i \bar{\jmath}}-P_{\bar{\imath} \bar{k} \bar{m}} \bar{P}_{j l n} g^{\bar{m} n}
$$

which is the usual constraint on the Riemann tensor of the special geometry. Note that by consistency of the left and right sides of (3.85), the one-form $P_{\bar{\imath} \bar{k}}$ must be a $(0,1)$-form, namely $P_{\bar{\imath} \bar{k}}=P_{\bar{\imath} \bar{k} l} d \bar{z}^{\bar{l}}$. The further Special Geometry constraints on the three tensor $\bar{P}_{i j k}$ are then consequences of equation (3.86), which implies:

$$
\begin{aligned}
\nabla_{[l} \bar{P}_{i] j k} & =0 \\
\nabla_{\bar{l}} \bar{P}_{i j k} & =0
\end{aligned}
$$

In particular, the first of equations (3.88) also implies that $\bar{P}_{i j k}$ is a completely symmetric tensor.

In summary, we have seen that the $N=2$ theory and the higher $N$ theories have essentially the same symplectic structure, the only difference being that since the scalar manifold of $N=2$ is not in general a coset manifold the symplectic structure allows the presence of a new geometrical quantity which physically corresponds to the anomalous magnetic moments of the $N=2$ theory. It goes without saying that, when $\mathcal{M}_{N=2}$ is itself a coset manifold [50], then the anomalous magnetic moments $\bar{P}_{i j k}$ must be expressible in terms of the vielbein of $G / H$. We give here two examples.

- Suppose $\mathcal{M}_{N=2}=\frac{S U(1,1)}{U(1)} \times \frac{O(2, n)}{O(2) \times O(n)}$. The symplectic sections entering the matrix $U$ can be written as follows:

$$
\begin{aligned}
& f=\mathrm{i} e^{\frac{\mathcal{K}}{2}}\left(L^{\Lambda}, L_{a}^{\Lambda}\right) \\
& h=\mathrm{i} e^{\frac{\mathcal{K}}{2}}\left(S L^{\Sigma} \eta_{\Lambda \Sigma}, \bar{S} L_{a}^{\Sigma} \eta_{\Lambda \Sigma}\right)
\end{aligned}
$$


where $\Lambda=1, \cdots, 2 n, a=3, \cdots, n, \eta_{\Lambda \Sigma}=(1,1,-1, \cdots,-1)$ and we have set $L^{\Lambda}=$ $\frac{1}{\sqrt{2}}\left(L_{1}^{\Lambda}+\mathrm{i} L_{2}^{\Lambda}\right)$. In particular from the pseudoorthogonality of $O(2, n)$ we have:

$$
L^{\Lambda} L^{\Sigma} \eta_{\Lambda \Sigma}=0
$$

Furthermore we have parametrized $\frac{S U(1,1)}{U(1)}$ as follows:

$$
M(S)=\frac{1}{\sqrt{\frac{4 \operatorname{ImS} S}{1+|S|^{2}+2 \operatorname{Im} S}}}\left(\begin{array}{cc}
\mathbb{1} & \frac{\mathrm{i}-S}{\mathrm{i}+S} \\
\frac{\mathrm{i}+\bar{S}}{\mathrm{i}-\bar{S}} & \mathbb{1}
\end{array}\right)
$$

We can then compute the embedded connection and vielbein using (3.55). In particular we find:

$$
\mathcal{P}=\left(\begin{array}{cc}
0 & P_{I} \\
P_{I} & -\bar{P} \delta_{a b}
\end{array}\right)
$$

We see that the general $P_{I \bar{J}}$ matrix in this case can be expressed in terms of the vielbein of $G / H$ and one finds that the only non vanishing anomalous magnetic moments are:

$$
\bar{P}_{a b S}=\delta_{a b} P_{, S}=e^{\mathcal{K}} \delta_{a b}
$$

- As a second example we consider the special coset manifold $\frac{S O^{*}(12)}{U(6)}$.

Note that this manifold also appears as the scalar manifold of the $D=4, N=6$ theory, and we refer the reader to section 4 for notations and parametrization of $G / H$.

The integrable system in this case can be written as follows:

$$
\nabla\left(\begin{array}{c}
V \\
V_{A B} \\
\bar{V} \\
\bar{V}^{A B}
\end{array}\right)=\left(\begin{array}{cccc}
0 & 0 & 0 & \frac{1}{2} P_{C D} \\
0 & 0 & P_{A B} & \frac{1}{2} P_{A B C D} \\
0 & \frac{1}{2} \bar{P}^{C D} & 0 & 0 \\
\bar{P}^{A B} & \frac{1}{2} P^{A B C D} & 0 & 0
\end{array}\right)\left(\begin{array}{c}
V \\
V_{C D} \\
\bar{V} \\
\bar{V}^{C D}
\end{array}\right)
$$

where $P^{A B C D}$ is the Kählerian vielbein $(1,0)$-form $\left(\bar{P}_{A B C D}=\left(P^{A B C D}\right)^{*}\right.$ is a $(0,1)-$ form) and:

$$
P_{A B}=\frac{1}{4 !} \epsilon_{A B C D E F} P^{C D E F} ; \quad \bar{P}^{A B}=\left(P_{A B}\right)^{*} .
$$

Moreover, $f_{A B}$ transforms in the $\mathbf{1 5}$ of $S U(6), f$ is an $S U(6)$ singlet and $\bar{f}^{A B}=$ $\left(f_{A B}\right)^{*}$. It follows:

$$
\nabla_{i}\left(\begin{array}{c}
V \\
V_{A B} \\
\bar{V} \\
\bar{V}^{A B}
\end{array}\right)=\left(\begin{array}{cccc}
0 & 0 & 0 & \frac{1}{2} P_{C D, i} \\
0 & 0 & P_{A B, i} & 0 \\
0 & 0 & 0 & 0 \\
0 & \frac{1}{2} P_{, i}^{A B C D} & 0 & 0
\end{array}\right)\left(\begin{array}{c}
V \\
V_{C D} \\
\bar{V} \\
\bar{V}^{C D}
\end{array}\right)
$$

Hence:

$$
\nabla_{i} \bar{V}^{A B}=\frac{1}{2} P_{, i}^{A B C D} V_{C D}
$$

If we set:

$$
V_{C D}=P_{C D}^{\bar{l}} V_{\bar{l}} ; \quad \bar{V}^{A B}=\bar{P}^{A B, k} V_{k}
$$


where the curved indices $k, \bar{l}$ are raised and lowered with the Kähler metric, one easily obtains:

$$
\nabla_{i} V_{j}=\frac{1}{2} P_{A B, i} P_{C D, j} P_{, k}^{A B C D} g^{k \bar{l}} f_{\bar{l}}=\frac{1}{4} \epsilon_{A B C D E F} P_{A B, i} P_{C D, j} P_{E F, k} g^{k \bar{l}} f_{\bar{l}} .
$$

Therefore the anomalous magnetic moment is given, in terms of the vielbein, as:

$$
\bar{P}_{i j k}=\frac{1}{4} \epsilon_{A B C D E F} P_{A B, i} P_{C D, j} P_{E F, k}
$$

The other two equations of the integral system give:

$$
\begin{gathered}
\nabla_{i} V=\frac{1}{2} P_{C D, i} V^{C D} \quad \rightarrow \quad \nabla_{i} V=V_{i} \\
\nabla_{i} V_{A B}=P_{A B, i} \bar{V} \rightarrow \nabla_{i} V_{\bar{j}}=g_{i \bar{j}} \bar{V}
\end{gathered}
$$

which are the remaining equations defining $N=2$ Special Geometry.

To complete the analogy between the $N=2$ theory and the higher $N$ theories in $D=4$, we also give for completeness, in the $N=2$ case, the central and matter charges, the differential relations among them and the sum rules. (Note that in $D=4 N=2$ we also have supersymmetric matter given by hypermultiplets whose scalar manifold is quaternionic. Since we are concerned mainly with central and matter charges we will not consider the coupling of the hypermultiplets here).

Let us note that also in $N=2$ the kinetic matrix $\mathcal{N}_{\Lambda \Sigma}$ which appears in the vector kinetic Lagrangian to:

$$
\begin{gathered}
\mathcal{L}_{\text {Kin }}^{(\text {vector })}=\mathrm{i} \overline{\mathcal{N}}_{\Lambda \Sigma} F_{\mu \nu}^{-\Lambda} F^{-\Sigma \mu \nu}+\text { h.c. } \\
F^{ \pm \Lambda}=\frac{1}{2}\left(\mathbb{1} \pm \mathrm{i}^{\star}\right) \mathrm{F}^{\Lambda} \\
\mathcal{G}_{\Lambda}^{-}=\overline{\mathcal{N}}_{\Lambda \Sigma} F_{\Lambda}^{-}
\end{gathered}
$$

is given in terms of $f, h$ by the formula:

$$
\mathcal{N}_{\Lambda \Sigma}=h_{\Lambda I}\left(f^{-1}\right)_{\Sigma}^{I}
$$

The columns of the matrix $f$ appear in the supercovariant electric field strength $\widehat{F}^{\Lambda}$ :

$$
\widehat{F}^{\Lambda}=F^{\Lambda}+f^{\Lambda} \bar{\psi}^{A} \psi^{B} \epsilon_{A B}-\mathrm{i} \bar{f}_{\bar{\imath}}^{\Lambda} \bar{\lambda}_{A}^{\bar{\imath}} \gamma_{a} \psi_{B} \epsilon_{A B} V^{a}+\text { h.c. }
$$

(The columns of $h_{I}^{\Lambda}$ would appear in the dual theory written in terms of the dual magnetic field strengths) .

The transformation laws for the chiral gravitino $\psi_{A}$ and gaugino $\lambda^{i A}$ fields are:

$$
\begin{gathered}
\delta \psi_{A \mu}=\mathcal{D}_{\mu} \epsilon_{A}+\epsilon_{A B} T_{\mu \nu} \gamma^{\nu} \epsilon^{B}+\cdots \\
\delta \lambda^{i A}=\mathrm{i} \partial_{\mu} z^{i} \gamma^{\mu} \epsilon^{A}+\frac{\mathrm{i}}{2} T_{\bar{\jmath} \mu \nu} \gamma^{\mu \nu} g^{i \bar{\jmath}} \epsilon^{A}+\cdots
\end{gathered}
$$

\footnotetext{
4 The same normalization for the vector kinetic lagrangian will be used in section 4 when discussing $D=4, N>2$ theories
} 
where:

$$
\begin{aligned}
T & \equiv h_{\Lambda} F^{\Lambda}-f^{\Lambda} \mathcal{G}_{\Lambda} \\
T_{\bar{\imath}} & \equiv \bar{h}_{\Lambda_{\bar{\imath}}} F^{\Lambda}-\bar{f}_{\bar{\imath}}^{\Lambda} \mathcal{G}_{\Lambda}
\end{aligned}
$$

are respectively the graviphoton and the matter-vectors $z^{i}(i=1, \cdots, n)$ are the complex scalar fields and the position of the $S U(2)$ automorphism index A $(\mathrm{A}, \mathrm{B}=1,2)$ is related to chirality (namely $\left(\psi_{A}, \lambda^{i A}\right)$ are chiral, $\left(\psi^{A}, \lambda_{A}^{\bar{\imath}}\right)$ antichiral). In principle only the (anti) self dual part of $F$ and $\mathcal{G}$ should appear in the transformation laws of the (anti)chiral fermi fields; however, exactly as in eqs. (3.49), (3.50) for $N>2$ theories, from equations (3.104), (3.105) it follows that :

$$
T^{+}=h_{\Lambda} F^{+\Lambda}-f^{\Lambda} \mathcal{G}_{\Lambda}^{+}=0
$$

so that $T=T^{-}$(and $\bar{T}=\bar{T}^{+}$). Note that both the graviphoton and the matter vectors are $U s p(n, n)$ invariant according to the fact that the fermions do not transform under the duality group (except for a possible R-symmetry phase). To define the physical charges let us note that in presence of electric and magnetic sources we can write:

$$
\int_{S^{2}} F^{\Lambda}=g^{\Lambda}, \quad \int_{S^{2}} \mathcal{G}_{\Lambda}=e_{\Lambda}
$$

The central charges and the matter charges are now defined as the integrals over a $S^{2}$ of the physical graviphoton and matter vectors:

$$
Z=\int_{S^{2}} T=\int_{S^{2}}\left(h_{\Lambda} F^{\Lambda}-f^{\Lambda} \mathcal{G}_{\Lambda}\right)=\left(h_{\Lambda}(z, \bar{z}) g^{\Lambda}-f^{\Lambda}(z, \bar{z}) e_{\Lambda}\right)
$$

where $z^{i}, \bar{z}^{\bar{\imath}}$ denote the v.e.v. of the moduli fields in a given background. Owing to eq (3.74) we get immediately:

$$
Z_{i}=\nabla_{i} Z
$$

We observe that if in a given background $Z_{i}=0$ the BPS states in this configuration have a minimum mass. Indeed

$$
\nabla_{i} Z=0 \rightarrow \partial_{i}|Z|^{2}=0 .
$$

As a consequence of the symplectic structure, one can derive two sum rules for $Z$ and $Z_{i}$ :

$$
|Z|^{2} \pm\left|Z_{i}\right|^{2} \equiv|Z|^{2} \pm Z_{i} g^{i \bar{\jmath} \bar{Z}_{\bar{\jmath}}}=-\frac{1}{2} P^{t} \mathcal{M}_{ \pm} P
$$

where:

$$
\begin{aligned}
\mathcal{M}_{+} & =\left(\begin{array}{cc}
\mathbb{1} & 0 \\
-\operatorname{Re} \mathcal{N} & \mathbb{1}
\end{array}\right)\left(\begin{array}{cc}
\operatorname{Im} \mathcal{N} & 0 \\
0 & \operatorname{Im} \mathcal{N}^{-1}
\end{array}\right)\left(\begin{array}{cc}
\mathbb{1} & -\operatorname{Re} \mathcal{N} \\
0 & \mathbb{1}
\end{array}\right) \\
\mathcal{M}_{-} & =\left(\begin{array}{cc}
\mathbb{1} & 0 \\
-\operatorname{Re} F & \mathbb{1}
\end{array}\right)\left(\begin{array}{cc}
\operatorname{Im} F & 0 \\
0 & \operatorname{Im} F^{-1}
\end{array}\right)\left(\begin{array}{cc}
\mathbb{1} & -\operatorname{Re} F \\
0 & \mathbb{1}
\end{array}\right)
\end{aligned}
$$

and:

$$
P=\left(g^{\Lambda}, e_{\Lambda}\right)
$$

Equation (3.117) is obtained by using exactly the same procedure as in (3.66). The sum rule (3.118) involves a matrix $\mathcal{M}_{-}$, which has exactly the same form as $\mathcal{M}_{+}$provided we perform the substitution $\mathcal{N}_{\Lambda \Sigma} \rightarrow F_{\Lambda \Sigma}=\frac{\partial^{2} F}{\partial X^{\Lambda} \partial X^{\Sigma}}\left(X^{\Lambda}=e^{-\frac{K}{2}} f^{\Lambda}\right)$. It can be derived 
in an analogous way by observing that, when a prepotential $F=F(X)$ exists, Special Geometry gives the following extra identity:

$$
f_{I}^{\Lambda}(F-\bar{F})_{\Lambda \Sigma} f_{J}^{\Sigma}=-\mathrm{i} \eta_{I J} \quad \eta=\left(\begin{array}{cc}
-1 & 0 \\
0 & \mathbb{1}_{n \times n}
\end{array}\right)
$$

from which it follows:

$$
\begin{aligned}
& f \eta f^{\dagger}=\mathrm{i}(F-\bar{F})^{-1} \\
& h \eta h^{\dagger}=\mathrm{i}\left(\bar{F}^{-1}-F^{-1}\right)^{-1} \equiv \mathrm{i} \bar{F}(F-\bar{F})^{-1} F
\end{aligned}
$$

Note that while $\operatorname{Im\mathcal {N}}$ has a definite (negative) signature, $\operatorname{ImF}$ is not positive definite.

\section{$4 \quad N>2$ four dimensional supergravities revisited}

In this section and in the following ones we apply the general considerations of section 3 to the various ungauged supergravity theories with scalar manifold $G / H$ for any $D$ and $N$. This excludes $D=4 N=2$, already discussed in section 3.2 , and $D=5 N=2$ for which we refer to the literature. Our aim is to write down the group theoretical structure of each theory, their symplectic or orthogonal embedding, the vector kinetic matrix, the supersymmetric transformation laws, the structure of the central and matter charges, the differential relations originating from the Maurer-Cartan equations and the sum rules they satisfy. For each theory we give the group-theoretical assignments for the fields, their supersymmetry transformation laws, the $(p+2)$-forms kinetic metrics and the relations satisfied by central and matter charges. As far as the boson transformation rules are concerned we prefer to write down the supercovariant definition of the field strengths (denoted by a superscript hat), from which the susy-laws are immediately retrieved. As it has been mentioned in section 3 it is here that the symplectic section $\left(f_{A B}^{\Lambda}, f_{I}^{\Lambda}, \bar{f}_{A B}^{\Lambda}, \bar{f}_{I}^{\Lambda}\right)$ appear as coefficients of the bilinear fermions in the supercovariant field-strengths while the analogous symplectic section $\left(h_{\Lambda A B}, h_{\Lambda I}, \bar{h}_{\Lambda A B}, \bar{h}_{\Lambda I}\right)$ would appear in the dual magnetic theory. We include in the supercovariant field-strengths also the supercovariant vielbein of the $G / H$ manifolds. Again this is equivalent to giving the susy transformation laws of the scalar fields. The dressed field strengths from which the central and matter charges are constructed appear instead in the susy transformation laws of the fermions for which we give the expression up to trilinear fermion terms. It should be stressed that the numerical coefficients in the aforementioned susy transformations and supercovariant field strengths are fixed by supersymmetry (or ,equivalently, by Bianchi identities in superspace ), but we have not worked out the relevant computations being interested in the general structure rather that in the precise numerical expressions. However the numerical factors could also be retrieved by comparing our formulae with those written in the standard literature on supergravity and performing the necessary redefinitions. The same kind of considerations apply to the central and matter charges whose precise normalization has not been fixed. In the Tables of the present and of the following sections, we give the group assignements for the supergravity fields; in particular, we quote the representation $R_{H}$ under which the scalar fields of the linearized theory (or the vielbein of $G / H$ of the full theory) transform. Furthermore, in general only the left-handed fermions (when they exist) are quoted. 
Right handed fermions transform in the complex conjugate representation of $H$. In the present section we apply the cosiderations given in section 3.2 to the $4 \mathrm{D}$-Supergravities for $N>2$. Throughout the section we denote by $A, B, \cdots$ indices of $S U(N), S U(N) \otimes U(1)$ being the automorphism group of the $N$-extended supersymmetry algebra. Lower and upper $S U(N)$ indices on the fermion fields are related to their left or right chirality respectively. If some fermion is a $S U(N)$ singlet chirality is denoted by the usual (L) or (R) suffixes. Right-handed fermions of $D=4$ transform in the complex f representation of $S U(N) \times U(1) \times H_{\text {matter }}$.

Furthermore for any boson field $v$ carrying $S U(N)$ indices we have that lower and upper indices are related by complex conjugation, namely:

$$
v_{A B \cdots}=\bar{v}^{A B \cdots}
$$

- Let us first consider the $N=3$ case 47. The coset space is:

$$
G / H=\frac{S U(3, n)}{S U(3) \otimes S U(n) \otimes U(1)}
$$

and the field content is given by:

$$
\begin{array}{lrr}
\left(V_{\mu}^{a}, \psi_{A \mu}, A_{\mu}, \chi_{(L)}\right) & A=1,2,3 \quad \text { (gravitational multiplet) } \\
\left(A_{\mu}, \lambda_{A}, \lambda_{(R)}, L(z, \bar{z})\right)^{I} & I=1, \cdots, n \quad \text { (vector multiplets) }
\end{array}
$$

The transformation properties of the fields are given in the following Table 11 The

Table 1: Transformation properties of fields in $D=4, N=3$

\begin{tabular}{|c||c|c|c|c|c|c|c|c|c|}
\hline & $V_{\mu}^{a}$ & $\psi_{A \mu}$ & $A_{\mu}^{\Lambda}$ & $\chi_{(L)}$ & $\lambda_{A}^{I}$ & $\lambda_{(L)}^{I}$ & $L_{A B}^{\Lambda}$ & $L_{I}^{\Lambda}$ & $R_{H}$ \\
\hline \hline$S U(3, n)$ & 1 & 1 & $3+n$ & 1 & 1 & 1 & $3+n$ & $3+n$ & - \\
\hline$S U(3)$ & 1 & 3 & 1 & 1 & 3 & 1 & 3 & 1 & 3 \\
\hline$S U(n)$ & 1 & 1 & 1 & 1 & $n$ & $n$ & 1 & $n$ & $n$ \\
\hline$U(1)$ & 0 & $\frac{n}{2}$ & 0 & $3 \frac{n}{2}$ & $3+\frac{n}{2}$ & $-3\left(1+\frac{n}{2}\right)$ & $n$ & -3 & $3+n$ \\
\hline
\end{tabular}

embedding of $S U(3, n)$ in $U s p(3+n, 3+n)$ allows to express the section $(f, h)$ in terms of $L$ as follows:

$$
\begin{aligned}
f_{\Sigma}^{\Lambda} & \equiv\left(L_{A B}^{\Lambda}, \bar{L}_{I}^{\Lambda}\right) \\
h_{\Lambda \Sigma} & =\mathrm{i}(J f J)_{\Lambda \Sigma}
\end{aligned} \quad J=\left(\begin{array}{cc}
\mathbb{1}_{3 \times 3} & 0 \\
0 & -\mathbb{1}_{n \times n}
\end{array}\right)
$$

where $A B$ are antisymmetric $S U(3)$ indices, $I$ is an index of $S U(n) \otimes U(1)$ and $\bar{L}_{I}^{\Lambda}$ denotes the complex conjugate of the coset representative. Using eq.s (4.5) and (4.6) we have:

$$
\mathcal{N}_{\Lambda \Sigma}=\left(h f^{-1}\right)_{\Lambda \Sigma}=\frac{1}{2} L_{\Lambda}^{A B} L_{A B \Sigma}+L_{\Lambda I} \bar{L}_{I \Sigma}
$$

\footnotetext{
${ }^{5}$ We recall that $R_{H}$ denotes the representation which the vielbein of the scalar manifold belongs to.
} 
The supercovariant field-strengths and the supercovariant scalar vielbein $\widehat{P}_{I}^{A}=$ $\left(L^{-1} \nabla^{(H)} L\right)_{I}^{A}$ are:

$$
\begin{aligned}
\widehat{F}^{\Lambda} & =d A^{\Lambda}-\frac{1}{2} f_{A B}^{\Lambda} \bar{\psi}^{A} \psi^{B}+\frac{\mathrm{i}}{2} f_{I}^{\Lambda} \bar{\lambda}_{A}^{I} \gamma_{a} \psi^{A} V^{a}+\mathrm{i} f_{A B}^{\Lambda} \bar{\chi}_{(R)} \gamma_{a} \psi_{C} \epsilon^{A B C} V^{a} \\
& + \text { h.c. } \\
\widehat{P}_{I}^{A} & =P_{I}^{A}-\bar{\lambda}_{B}^{I} \psi_{C} \epsilon^{A B C}-\bar{\lambda}_{I(R)} \psi^{A}
\end{aligned}
$$

where:

$$
\begin{aligned}
P^{I A} & =\frac{1}{2} \epsilon^{A B C} P_{I B C}=\frac{1}{2} \epsilon^{A B C}\left(L^{-1} \nabla^{(S U(3) \times U(1))} L\right)_{I B C} \\
& =P_{, i}^{I A} d z^{i} \\
\bar{P}^{I A} & =P_{I A}
\end{aligned}
$$

$z^{i}$ being the (complex) coordinates of $G / H$ and $H=H_{A u t}=S U(3) \times U(1)$. The chiral fermions transformation laws are given by:

$$
\begin{aligned}
\delta \psi_{A} & =\nabla \epsilon_{A}+2 \mathrm{i} T_{A B \mid a b}^{-} \Delta^{a b c} V_{c} \epsilon^{B}+\cdots \\
\delta \chi_{(L)} & =1 / 2 T_{A B \mid a b}^{-} \gamma^{a b} \epsilon_{C} \epsilon^{A B C}+\cdots \\
\delta \lambda_{A}^{I} & =-\mathrm{i} P_{, i}^{I B} \partial_{a} z^{i} \gamma^{a} \epsilon^{C} \epsilon_{A B C}+T_{I \mid a b} \gamma^{a b} \epsilon_{A}+\cdots \\
\delta \lambda_{(L)}^{I} & =\mathrm{i} P_{, i}^{I A} \partial_{a} z^{i} \gamma^{a} \epsilon_{A}+\cdots
\end{aligned}
$$

where $T_{A B}$ and $T_{I}$ have the general form given in equation (3.48). Therefore, the general form of the dyonic charges $\left(Z_{A B}, Z_{I}\right)$ are given by eqns. (3.51)- (3.53). From the general form of the Maurer-Cartan equations for the embedded coset representatives $U \in U \operatorname{sp}(n, n)$, we find:

$$
\nabla^{(H)}\left(\begin{array}{c}
f_{A B}^{\Lambda} \\
h_{\Lambda A B}
\end{array}\right)=\left(\begin{array}{c}
\bar{f}_{I}^{\Lambda} \\
\bar{h}_{\Lambda I}
\end{array}\right) P_{I}^{C} \epsilon_{A B C}
$$

According to the discussion given in section 3, using (3.51), (3.52) one finds:

$$
\begin{aligned}
\nabla^{(H)} Z_{A B} & =\bar{Z}^{I} P_{I}^{C} \epsilon_{A B C} \\
\nabla^{(H)} Z_{I} & =\frac{1}{2} \bar{Z}^{A B} P_{I}^{C} \epsilon_{A B C}
\end{aligned}
$$

and the sum rule:

$$
\frac{1}{2} Z^{A B} Z_{A B}+Z_{I} \bar{Z}_{I}=-\frac{1}{2} P^{t} \mathcal{M}(\mathcal{N}) P
$$

where the matrix $\mathcal{M}(\mathcal{N})$ has the same form as in equation (3.66) in terms of the kinetic matrix $\mathcal{N}$ of eq. (4.7) and $P$ is the charge vector $P^{t}=(g, e)$.

- For $N=4$ 48, the coset space is a product:

$$
G / H=\frac{S U(1,1)}{U(1)} \otimes \frac{O(6, n)}{O(6) \otimes O(n)}
$$


The field content is given by: Gravitational multiplet:

$$
\left(V_{\mu}^{a}, \psi_{A \mu}, A_{\mu}^{A B}, \chi_{A B C}, S\right) \quad(A, B=1, \cdots, 4)
$$

Vector multiplets:

$$
\left(A_{\mu}, \lambda^{A}, 6 \phi\right)^{I} \quad(I=1, \cdots, n)
$$

The coset representative can be written as:

$$
L_{\Sigma}^{\Lambda} \rightarrow M(S) L_{\Sigma}^{\Lambda}
$$

where $L_{\Sigma}^{\Lambda}$ parametrizes the coset manifold $\frac{O(6, n)}{O(6) \otimes O(n)}$ and

$$
M(S)=\frac{1}{\sqrt{\frac{4 I m S}{1+|S|^{2}+2 \operatorname{ImS}}}}\left(\begin{array}{cc}
\mathbb{1} & \frac{\mathrm{i}-S}{\mathrm{i}+S} \\
\frac{\mathrm{i}+\bar{S}}{\mathrm{i}-\bar{S}} & \mathbb{1}
\end{array}\right)
$$

The group assignments of the fields are given in Table 2. With the given coset

Table 2: $D=4, N=4$ transformation properties

\begin{tabular}{|c||c|c|c|c|c|c|c|c|}
\hline & $V_{\mu}^{a}$ & $\psi_{A \mid \mu}$ & $A_{\mu}^{\Lambda}$ & $\chi_{A B C}$ & $\lambda_{I A}$ & $M(S) L_{A B}^{\Lambda}$ & $M(S) L_{I}^{\Lambda}$ & $R_{H}$ \\
\hline \hline$S U(1,1)$ & 1 & 1 & - & 1 & 1 & $2 \times 1$ & $2 \times 1$ & - \\
\hline$O(6, n)$ & 1 & 1 & $6+n$ & 1 & 1 & $1 \times(6+n)$ & $1 \times(6+n)$ & - \\
\hline$O(6)$ & 1 & 4 & 1 & $\overline{4}$ & $\overline{4}$ & $1 \times 6$ & 1 & 6 \\
\hline$O(n)$ & 1 & 1 & 1 & 1 & $n$ & 1 & $n$ & $n$ \\
\hline$U(1)$ & 1 & $\frac{1}{2}$ & 1 & $\frac{3}{2}$ & $-\frac{1}{2}$ & 1 & 1 & 0 \\
\hline
\end{tabular}

parametrizations the symplectic embedded section $\left(f_{\Sigma}^{\Lambda}, h_{\Lambda \Sigma}\right)$ is (apart from a unessential phase $\left.\frac{\mathrm{i}+S}{\mathrm{i}-\bar{S}}\right)$ :

$$
\begin{aligned}
f_{\Sigma}^{\Lambda} & =\mathrm{i} e^{\frac{K}{2}}\left(L_{A B}^{\Lambda}, L_{I}^{\Lambda}\right) \\
h_{\Lambda \Sigma} & =\mathrm{i} e^{\frac{K}{2}}\left(S L_{A B}^{\Gamma} \eta_{\Lambda \Gamma}, \bar{S} L^{\Gamma}{ }_{I} \eta_{\Lambda \Gamma}\right)
\end{aligned}
$$

where $K=-\log [\mathrm{i}(S-\bar{S})]$ is the Kähler potential of $\frac{S U(1,1)}{U(1)}$, and the kinetic matrix $\mathcal{N}=h f^{-1}$ takes the form:

$$
\mathcal{N}_{\Lambda \Sigma}=\frac{1}{2}(S-\bar{S}) \bar{L}_{\Lambda}^{A B} L_{\Sigma A B}+\bar{S} \eta_{\Lambda \Sigma}
$$

The supercovariant field strengths and the vielbein of the coset manifold are:

$$
\begin{aligned}
\widehat{F}^{\Lambda} & =d A^{\Lambda}+\left[f_{A B}^{\Lambda}\left(c_{1} \bar{\psi}^{A} \psi^{B}+c_{2} \bar{\psi}_{C} \gamma_{a} \chi^{A B C} V^{a}\right)\right. \\
& \left.+f_{I}^{\Lambda}\left(c_{3} \bar{\psi}^{A} \gamma_{a} \lambda_{A}^{I} V^{a}+c_{4} \bar{\chi}^{A B C} \gamma_{a b} \lambda^{I D} \epsilon_{A B C D} V^{a} V^{b}\right)+\text { h.c. }\right] \\
\widehat{P} & =P-\bar{\psi}^{A} \chi^{B C D} \epsilon_{A B C D} \\
\widehat{P}_{A B}^{I} & =P_{A B}^{I}-\left(\bar{\psi}_{A} \lambda_{B}^{I}+\epsilon_{A B C D} \bar{\psi}^{C} \lambda^{I D}\right)
\end{aligned}
$$


where $P=P_{, S} d S$ and $P_{A B}^{I}=P_{A B, i}^{I} d \phi^{i}$ are the vielbein of $\frac{S U(1,1)}{U(1)}$ and $\frac{O(6, n)}{O(6) \times O(n)}$ respectively. The fermion transformation laws are:

$$
\begin{aligned}
\delta \psi_{A} & =\nabla \epsilon_{A}+a_{1} T_{A B \mid a b}^{-} \Delta^{a b c} \epsilon^{B} V_{c}+\cdots \\
\delta \chi_{A B C} & =a_{2} P_{, S} \partial_{a} S \gamma^{a} \epsilon^{D} \epsilon_{A B C D}+a_{3} T_{[A B \mid a b}^{-} \gamma^{a b} \epsilon_{C]}+\cdots \\
\delta \lambda_{A}^{I} & =a_{3} P_{A B, i}^{I} \partial_{a} \phi^{i} \gamma^{a} \epsilon^{B}+a_{4} T_{a b}^{-I} \gamma^{a b} \epsilon_{A}+\cdots
\end{aligned}
$$

where the 2-forms $T_{A B}$ and $T_{I}$ are defined in eq.(3.48) By integration of these twoforms, using eq.(3.50)-(3.53) we find the central and matter dyonic charges given in eq.s (3.51), (3.52). From the Maurer-Cartan equations for $f, h$ and the definitions of the charges one easily finds:

$$
\begin{aligned}
\nabla^{S U(4) \otimes U(1)} Z_{A B} & =\bar{Z}^{I} P_{I A B}+\frac{1}{2} \epsilon_{A B C D} \bar{Z}^{C D} P \\
\nabla^{S O(n)} Z_{I} & =\frac{1}{2} \bar{Z}^{A B} P_{I A B}+Z_{I} \bar{P}
\end{aligned}
$$

In terms of the kinetic matrix (4.27) the sum rule for the charges is given by eqs.(3.65)-(3.67):

$$
\frac{1}{2} Z_{A B} \bar{Z}^{A B}+Z_{I} \bar{Z}_{I}=-\frac{1}{2} P^{t} \mathcal{M}(\mathcal{N}) P
$$

For $N>4$ the only available supermultiplet is the gravitational one, so that $H_{\text {matter }}=\mathbb{1}$. The embedding procedure is much simpler than in the matter coupled supergravities since for each $N>4$ there exists a representation of the scalar manifold isometry group $G$ given in terms of $U s p\left(n_{v}, n_{v}\right)$ matrices.

- For the $N=5$ theory [51] the coset manifold is:

$$
G / H=\frac{S U(1,5)}{U(5)}
$$

The field content and the group assignments are displayed in table 3 . Here $x, y, \cdots=$

Table 3: Transformation properties of fields in $D=4, N=5$

\begin{tabular}{|c||c|c|c|c|c|c|}
\hline & $V^{a}$ & $\psi_{A} ;$ & $\chi_{A B C}, \chi_{L}$ & $A^{\Lambda \Sigma}$ & $L_{A}^{x}$ & $R_{H}$ \\
\hline \hline$S U(1,5)$ & 1 & 1 & 1 & - & 6 & - \\
\hline$S U(5)$ & 1 & 5 & $10+1$ & 1 & 5 & $\overline{5}$ \\
\hline$U(1)$ & 0 & $\frac{1}{2}$ & $\left(\frac{3}{2},-\frac{5}{2}\right)$ & 0 & 1 & 2 \\
\hline
\end{tabular}

$1, \cdots, 6$ and $A, B, C \cdots=1, \cdots, 5$ are indices of the fundamental representations of $S U(1,5)$ and $S U(5)$,respectively. $L_{A}^{x}$ denote as usual the coset representative in the fundamental representation of $S U(1,5)$. The antisymmetric couple $\Lambda \Sigma$, $\Lambda, \Sigma=1, \cdots, 5$, enumerates the ten vectors. The embedding of $S U(1,5)$ into the Gaillard-Zumino group $U s p(10,10)$ is given in terms of the three-times antisymmetric representation of $S U(1,5)$, a generic element $t^{x y z}$ satisfying:

$$
t^{x y z}=\frac{1}{3 !} \epsilon^{x y z u v w} t_{u v w}
$$


We may decompose $t^{x y z}$ as follows:

$$
t^{x y z}=\left(\begin{array}{c}
t^{\Lambda \Sigma 6} \\
t^{\Lambda \Sigma \Gamma}=\epsilon^{\Lambda \Sigma \Gamma \Delta \Pi 6} t_{\Delta \Pi 6}
\end{array}\right) \quad(\Lambda, \Sigma, \cdots=1, \cdots, 5)
$$

In the following we write $t^{\Lambda \Sigma 6} \equiv t^{\Lambda \Sigma}$. The 20 dimensional vector $\left(F^{\mp \Lambda \Sigma}, \mathcal{G}_{\Lambda \Sigma}^{\mp}\right)$ transforms under $S p(20, \mathbb{R})$, while, for fixed $A B$, each of the 20-dimensional vectors $\left(f_{A B}^{\Lambda \Sigma}, h_{\Lambda \Sigma A B}\right)$ of the embedding matrix:

$$
U=\frac{1}{\sqrt{2}}\left(\begin{array}{ll}
f+\mathrm{i} h & \bar{f}+\mathrm{i} \bar{h} \\
f-\mathrm{i} h & \bar{f}-\mathrm{i} \bar{h}
\end{array}\right)
$$

transforms under $U s p(10,10)$.

The supercovariant field-strengths and vielbein are:

$$
\begin{aligned}
\widehat{F}^{\Lambda \Sigma} & =d A^{\Lambda \Sigma}+\left(f_{A B}^{\Lambda \Sigma}\left(a_{1} \bar{\psi}^{A} \psi^{B}+a_{2} \bar{\psi}_{C} \gamma_{a} \chi^{A B C} V^{a}\right)+\text { h.c. }\right) \\
\widehat{P}_{A B C D} & =P_{A B C D}-\bar{\chi}_{[A B C} \psi_{D]}-\epsilon_{A B C D E} \bar{\chi}^{(R)} \psi^{E}
\end{aligned}
$$

where $P_{A B C D}=\epsilon_{A B C D F} P^{F}$ is the complex vielbein, completely antisymmetric in $S U(5)$ indices and $\left(P_{A B C D}\right)^{\star}=\bar{P}^{A B C D}$.

The fermion transformation laws are:

$$
\begin{aligned}
\delta \psi_{A} & =\nabla \epsilon_{A}+a_{3} T_{A B \mid a b}^{-} \Delta^{a b c} \epsilon^{B} V_{c}+\cdots \\
\delta \chi_{A B C} & =a_{4} P_{A B C D, i} \partial_{a} \phi^{i} \gamma^{a} \epsilon^{D}+a_{5} T_{[A B \mid a b}^{-} \gamma^{a b} \epsilon_{C]}+\cdots \\
\delta \chi_{(L)} & =a_{6} \bar{P}_{, \bar{i}}^{A B C D} \partial_{a} \phi^{i} \gamma^{a} \epsilon^{E} \epsilon_{A B C D E}+\cdots
\end{aligned}
$$

where:

$$
\begin{aligned}
T_{A B} & =-\frac{\mathrm{i}}{2}\left(\bar{f}^{-1}\right)_{\Lambda \Sigma A B} F^{\Lambda \Sigma}=\frac{1}{4}(\mathcal{N}-\overline{\mathcal{N}})_{\Lambda \Sigma, \Gamma \Delta} f_{{ }_{A B}^{\Gamma \Delta}} F^{\Lambda \Sigma} \\
& =\frac{1}{2}\left(h_{\Lambda \Sigma A B} F^{\Lambda \Sigma}-f_{A B}^{\Lambda \Sigma} \mathcal{G}_{\Lambda \Sigma}\right) \\
\mathcal{N}_{\Lambda \Sigma, \Delta \Pi} & =\frac{1}{2} h_{\Lambda \Sigma \mid A B}\left(f^{-1}\right)_{\Delta B}^{A B} \\
\mathcal{G}_{\Lambda \Sigma} & =-\mathrm{i} / 2 \frac{\partial \mathcal{L}}{\partial F^{\Lambda \Sigma}}
\end{aligned}
$$

With a by now familiar procedure one finds the following (complex) central charges:

$$
Z_{A B}=\frac{1}{2}\left(h_{\Lambda \Sigma \mid A B} g^{\Lambda \Sigma}-f_{A B}^{\Lambda \Sigma} e_{\Lambda \Sigma}\right)
$$

where:

$$
\begin{aligned}
g^{\Lambda \Sigma} & =\int_{S^{2}} F^{\Lambda \Sigma} \\
e_{\Lambda \Sigma} & =\int_{S^{2}} \mathcal{G}_{\Lambda \Sigma}
\end{aligned}
$$

From the Maurer-Cartan equation

$$
\nabla^{(U(5))} f_{\Lambda \Sigma \mid A B}=\frac{1}{2} \bar{f}_{\Lambda \Sigma}^{C D} P_{A B C D}
$$


and the analogous one for $h$ we find:

$$
\nabla^{(U(5))} Z_{A B}=\frac{1}{2} \bar{Z}^{C D} P_{A B C D}
$$

Finally, the sum rule for the central charges is:

$$
\frac{1}{2} Z_{A B} \bar{Z}^{A B}=-\frac{1}{2}\left(g^{\Lambda \Sigma}, e_{\Lambda \Sigma}\right) \mathcal{M}(\mathcal{N})_{\Lambda \Sigma, \Gamma \Delta}\left(\begin{array}{c}
g^{\Gamma \Delta} \\
e_{\Gamma \Delta}
\end{array}\right)
$$

where the matrix $\mathcal{M}(\mathcal{N})$ has exactly the same form as in eq (3.66).

- The scalar manifold of the $N=6$ theory has the coset structure:

$$
G / H=\frac{S O^{\star}(12)}{U(6)}
$$

We recall that $S O^{\star}(2 n)$ is defined as the subgroup of $O(2 n, \mathbb{C})$ that preserves the sesquilinear antisymmetric metric:

$$
L^{\dagger} C L=C, \quad C=\left(\begin{array}{cc}
0 & \mathbb{1} \\
-\mathbb{1} & 0
\end{array}\right)
$$

The field content and transformation properties are given in Table $\llbracket$ where $A, B, C=$

Table 4: Transformation properties of fields in $D=4, N=6$

\begin{tabular}{|c||c|c|c|c|c|c|}
\hline & $V^{a}$ & $\psi_{A}$ & $\chi_{A B C}, \chi_{A}$ & $A^{\Lambda}$ & $S_{r}^{\alpha}$ & $R_{H}$ \\
\hline \hline$S O^{\star}(12)$ & 1 & 1 & 1 & - & $\underline{32}$ & - \\
\hline$S U(6)$ & 1 & 6 & $(20+6)$ & 1 & $(15,1)+(\overline{15}, \overline{1})$ & $\overline{1} 5$ \\
\hline$U(1)$ & 0 & $\frac{1}{2}$ & $\left(\frac{3}{2},-\frac{5}{2}\right)$ & 0 & $(1,-3)+(-1,3)$ & 2 \\
\hline
\end{tabular}

$1, \cdots, 6$ are $S U(6)$ indices in the fundamental representation and $\Lambda=1, \cdots, 16$. As it happens in the $N=5$ theory, the $\underline{32}$ spinor representation of $S O^{\star}(12)$ can be given in terms of a $U s p(16,16)$ matrix, which we denote by $S_{r}^{\alpha}(\alpha, r=1, \cdots, 32)$, so that the embedding is automatically realized in terms of the spinor representation. Employing the usual notation we may set:

$$
S_{r}^{\alpha}=\frac{1}{\sqrt{2}}\left(\begin{array}{cc}
f_{I}^{\Lambda}+\mathrm{i} h_{\Lambda I} & \bar{f}_{I}^{\Lambda}+\mathrm{i} \bar{h}_{\Lambda I} \\
f_{I}^{\Lambda}-\mathrm{i} h_{\Lambda I} & \bar{f}_{I}^{\Lambda}-\mathrm{i} \bar{h}_{\Lambda I}
\end{array}\right)
$$

where $\Lambda, I=1, \cdots, 16$. With respect to $S U(6)$, the sixteen symplectic vectors $\left(f_{I}^{\Lambda}, h_{\Lambda I}\right),(I=1, \cdots, 16)$ are reducible into the antisymmetric 15 - dimensional representation plus a singlet of $S U(6)$ :

$$
\left(f_{I}^{\Lambda}, h_{\Lambda I}\right) \rightarrow\left(f_{A B}^{\Lambda}, h_{\Lambda A B}\right)+\left(f^{\Lambda}, h_{\Lambda}\right)
$$

It is precisely the existence of a $S U(6)$ singlet which allows for the Special Geometry structure of $\frac{S O^{*}(12)}{U(6)}$ as discussed in section 3.2 Note that the coset element $S_{r}^{\alpha}$ has 
no definite $U(1)$ weight since the submatrices $f_{A B}^{\Lambda}, f^{\Lambda}$ have the weights 1 and 3 respectively. The supercovariant field-strenghts and the coset manifold vielbein have the following expression:

$$
\begin{aligned}
\widehat{F}^{\Lambda} & =d A^{\Lambda}+\left[f_{A B}^{\Lambda}\left(a_{1} \bar{\psi}^{A} \psi^{B}+a_{2} \bar{\psi}_{C} \gamma_{a} \chi^{A B C} V^{a}\right)\right. \\
& \left.+a_{3} f^{\Lambda} \bar{\psi}_{C} \gamma_{a} \chi^{C} V^{a}+h . c .\right] \\
\widehat{P}_{A B C D} & =P_{A B C D}-\bar{\chi}_{[A B C} \psi_{D]}-\epsilon_{A B C D E F} \bar{\chi}^{E} \psi^{F}
\end{aligned}
$$

where $P_{A B C D}=P_{A B C D, i} d z^{i}$ is the Kähler vielbein of the coset. The fermion transformation laws are:

$$
\begin{aligned}
\delta \psi_{A} & =\nabla \epsilon_{A}+b_{1} T_{A B \mid a b}^{-} \Delta^{a b c} \epsilon^{B} V_{c}+\cdots \\
\delta \chi_{A B C} & =b_{2} P_{A B C D \mid a} \gamma^{a} \epsilon^{D}+b_{3} T_{[A B \mid a b}^{-} \gamma^{a b} \epsilon_{C]}+\cdots \\
\delta \chi_{A} & =b_{4} P^{B C D E \mid a} \gamma^{a} \epsilon^{F} \epsilon_{A B C D E F}+b_{5} T_{a b}^{-} \gamma^{a b} \epsilon_{A}+\cdots
\end{aligned}
$$

where:

$$
\begin{aligned}
T_{A B} & =-\mathrm{i}\left(\bar{f}^{-1}\right)_{\Lambda A B} F^{-\Lambda} \\
T & =-\mathrm{i}\left(\bar{f}^{-1}\right)_{\Lambda} F^{-\Lambda}
\end{aligned}
$$

With the usual procedure we have the following complex dyonic central charges:

$$
\begin{aligned}
Z_{A B} & =h_{\Lambda A B} g^{\Lambda}-f_{A B}^{\Lambda} e_{\Lambda} \\
Z & =h_{\Lambda} g^{\Lambda}-f^{\Lambda} e_{\Lambda}
\end{aligned}
$$

in the $\underline{15}$ and singlet representation of $S U(6)$ respectively. Notice that although we have 16 graviphotons, only 15 central charges are present in the supersymmetry algebra. The singlet charge plays a role analogous to a "matter" charge. From the Maurer-Cartan equations:

$$
\begin{aligned}
\nabla f_{A B}^{\Lambda} & =\frac{1}{2} \bar{f}^{\Lambda \mid C D} P_{A B C D}+\frac{1}{4 !} \bar{f}^{\Lambda} \epsilon_{A B C D E F} P^{C D E F} \\
\nabla f^{\Lambda} & =\frac{1}{2 ! 4 !} f^{\Lambda \mid A B} \epsilon_{A B C D E F} P^{C D E F}
\end{aligned}
$$

and the relation (3.42) one finds:

$$
\begin{aligned}
\nabla^{(U(6))} Z_{A B} & =\frac{1}{2} \bar{Z}^{C D} P_{A B C D}+\frac{1}{4 !} \bar{Z} \epsilon_{A B C D E F} P^{C D E F} \\
\nabla^{(U(1))} Z & =\frac{1}{2 ! 4 !} \bar{Z}^{A B} \epsilon_{A B C D E F} P^{C D E F}
\end{aligned}
$$

and the sum-rule (3.65):

$$
\frac{1}{2} Z_{A B} \bar{Z}^{A B}+Z \bar{Z}=-\frac{1}{2}\left(g^{\Lambda}, \quad e_{\Lambda}\right) \mathcal{M}(\mathcal{N})_{\Lambda \Sigma}\left(\begin{array}{c}
g^{\Sigma} \\
e_{\Sigma}
\end{array}\right)
$$

with the usual meaning for $\mathcal{M}(\mathcal{N})$ (see eq.(3.66)). 
Table 5: Field content and group assignments in $D=4, N=8$ supergravity

\begin{tabular}{|c||c|c|c|c|c|c|}
\hline & $V_{\mu}^{a}$ & $\psi_{A}$ & $A_{\mu}^{\Lambda \Sigma}$ & $\chi_{A B C}$ & $S_{r}^{\alpha}$ & $R_{H}$ \\
\hline \hline$E_{7(-7)}$ & 1 & 1 & - & 1 & 56 & - \\
\hline$S U(8)$ & 1 & 8 & 1 & 56 & $28+\overline{28}$ & 70 \\
\hline
\end{tabular}

- In the $N=8$ case [52 the coset manifold is:

$$
G / H=\frac{E_{7(-7)}}{S U(8)}
$$

The field content and group assignments are given in the following Table 5:

As in $N=5,6$, the embedding is automatically realized in terms of the $\underline{56}$ defining representation for $E_{7}$ which belongs to $U s p(28,28)$ and it is given by the usual coset element (3.38) where

$$
\begin{aligned}
f+\mathrm{i} h & \equiv f_{A B}^{\Lambda \Sigma}+\mathrm{i} h_{\Lambda \Sigma A B} \\
\bar{f}-\mathrm{i} \bar{h} & \equiv \bar{f}^{\Lambda \Sigma A B}-\mathrm{i} \bar{h}_{\Lambda \Sigma}^{A B}
\end{aligned}
$$

$\Lambda \Sigma, A B$ are couples of antisymmetric indices, with $\Lambda, \Sigma, A, B$ running from 1 to 8 . The supercovariant field-strengths and coset manifold vielbein are:

$$
\begin{aligned}
\widehat{F}^{\Lambda \Sigma} & =d A^{\Lambda \Sigma}+\left[f_{A B}^{\Lambda \Sigma}\left(a_{1} \bar{\psi}^{A} \psi^{B}+a_{2} \bar{\chi}^{A B C} \gamma_{a} \psi_{C} V^{a}\right)+\text { h.c. }\right] \\
\widehat{P}_{A B C D} & =P_{A B C D}-\bar{\chi}_{[A B C} \psi_{D]}+\text { h.c. }
\end{aligned}
$$

where $P_{A B C D}=\frac{1}{4 !} \epsilon_{A B C D E F G H} \bar{P}^{E F G H} \equiv\left(L^{-1} \nabla^{S U(8)} L\right)_{A B \mid C D}=P_{A B C D, i} d \phi^{i}\left(\phi^{i}\right.$ coordinates of $G / H)$. The fermion transformation laws are given by:

$$
\begin{aligned}
\delta \psi_{A} & =\nabla \epsilon_{A}+a_{3} T_{A B \mid a b}^{-} \Delta^{a b c} \epsilon^{B} V_{c}+\cdots \\
\delta \chi_{A B C} & =a_{4} P_{A B C D, a} \gamma^{a} \epsilon^{D}+a_{5} T_{[A B \mid a b}^{-} \gamma^{a b} \epsilon_{C]}+\cdots
\end{aligned}
$$

where:

$$
\begin{aligned}
T_{A B} & =-\frac{\mathrm{i}}{2}\left(\bar{f}^{-1}\right)_{\Lambda \Sigma A B} F^{\Lambda \Sigma}=\frac{1}{4}(\mathcal{N}-\overline{\mathcal{N}})_{\Lambda \Sigma, \Gamma \Delta} f_{A B}^{\Lambda \Sigma} F^{\Gamma \Delta} \\
& =\frac{1}{2}\left(h_{\Lambda \Sigma A B} F^{\Lambda \Sigma}-f_{A B}^{\Lambda \Sigma} \mathcal{G}_{\Lambda \Sigma}\right)
\end{aligned}
$$

with

$$
\begin{aligned}
\mathcal{N}_{\Lambda \Sigma, \Gamma \Delta} & =\frac{1}{2} h_{\Lambda \Sigma A B}\left(f^{-1}\right)_{\Gamma \Delta}^{A B} \\
\mathcal{G}_{\Lambda \Sigma} & =-\mathrm{i} / 2 \frac{\partial \mathcal{L}}{\partial F^{\Lambda \Sigma}}
\end{aligned}
$$

With the usual manipulations we obtain the central charges:

$$
Z_{A B}=\frac{1}{2}\left(h_{\Lambda \Sigma A B} g^{\Lambda \Sigma}-f_{A B}^{\Lambda \Sigma} e_{\Lambda \Sigma}\right)
$$


the differential relations:

$$
\nabla^{S U(8)} Z_{A B}=\frac{1}{2} \bar{Z}^{C D} P_{A B C D}
$$

and the sum rule:

$$
\frac{1}{2} Z_{A B} Z^{A B}=-\frac{1}{8}\left(g^{\Lambda \Sigma}, e_{\Lambda \Sigma}\right) \mathcal{M}(\mathcal{N})_{\Lambda \Sigma, \Gamma \Delta}\left(\begin{array}{c}
g^{\Gamma \Delta} \\
e_{\Gamma \Delta}
\end{array}\right)
$$

\section{Application of the previous formalism to extremal black-holes in four dimensions}

Recently, considerable progress has been made in the study of general properties of black holes arising in supersymmetric theories of gravity such as extended supergravities, string theory and M-theory [53]. Of particular interest are extremal black holes in four dimensions which correspond to BPS saturated states [54 and whose ADM mass depends, beyond the quantized values of electric and magnetic charges, on the asymptotic value of scalars at infinity. The latter describe the moduli space of the theory. Another physical relevant quantity, which depends only on quantized electric and magnetic charges, is the black hole entropy, which can be defined macroscopically, through the BekensteinHawking area-entropy relation or microscopically, through D-branes techniques [55] by counting of microstates [56]. It has been further realized that the scalar fields, independently of their values at infinity, flow towards the black hole horizon to a fixed value of pure topological nature given by a certain ratio of electric and magnetic charges [22]. These "fixed scalars" correspond to the extrema of the ADM mass in moduli space while the black-hole entropy is the actual value of the squared ADM mass at this point [23]. In theories with $N>2$, extremal black-holes preserving one supersymmetry have the further property that all central charge eigenvalues other than the one equal to the BPS mass flow to zero for "fixed scalars". The black-hole entropy is still given by the square of the ADM mass for "fixed scalars" 24]. Recently [57], the nature of these extrema has been further studied and shown that they generically correspond to non degenerate minima for $N=2$ theories whose relevant moduli space is the special geometry of $N=2$ vector multiplets. The entropy formula turns out to be in all cases a U-duality invariant expression (homogeneous of degree two) built out of electric and magnetic charges and as such can be in fact also computed through certain (moduli-independent) topological quantities which only depend on the nature of the U-duality groups and the appropriate

representations of electric and magnetic charges. For example, in the $N=8$ theory the entropy was shown to correspond to the unique quartic $E_{7}$ invariant built with its 56 dimensional representation [58.

\section{Central charges, U-invariants and entropy}

In $D=4$, extremal black-holes preserving one supersymmetry correspond to $N$-extended multiplets with

$$
M_{A D M}=\left|Z_{1}\right|>\left|Z_{2}\right| \cdots>\left|Z_{[N / 2]}\right|
$$


where $Z_{\alpha}, \alpha=1, \cdots,[N / 2]$, are the proper values of the central charge antisymmetric matrix written in normal form [59]. The central charges $Z_{A B}=-Z_{B A}, A, B=1, \cdots, N$, and matter charges $Z_{I}, I=1, \cdots, n$ are those (moduli-dependent) symplectic invariant combinations of field strenghts and their duals (integrated over a large two-sphere) which appear in the gravitino and gaugino supersymmetry variations respectively [60], 61], 20]. Note that the total number of vector fields is $n_{v}=N(N-1) / 2+n$ (with the exception of $N=6$ in which case there is an extra singlet graviphoton) 29.

It was shown in ref. [24] that at the attractor point, where $M_{A D M}$ is extremized, supersymmetry requires that $Z_{\alpha}, \alpha>1$, vanish together with the matter charges $Z_{I}, I=1, \cdots, n$ ( $n$ is the number of matter multiplets, which can exist only for $N=3,4$ )

This result can be used to show that for "fixed scalars", corresponding to the attractor point, the scalar "potential" of the geodesic action [62 [57]

$$
V=-\frac{1}{2} P^{t} \mathcal{M}(\mathcal{N}) P
$$

is extremized in moduli space. Here, we recall that $P$ is the symplectic vector $P=\left(p^{\Lambda}, q_{\Lambda}\right)$ of quantized electric and magnetic charges introduced in eqn. (3.67) and $\mathcal{M}(\mathcal{N})$ is the $2 n_{v} \times 2 n_{v}$ symplectic matrix given in eqn. (3.66).

The above assertion is the result of computing the extremum of (6.88) by use of equations (3.63), (3.65). We obtain:

$$
P^{A B C D} Z_{A B} Z_{C D}=0 ; \quad Z_{I}=0
$$

$P_{A B C D}$ being the vielbein of the scalar manifold, completely antisymmetric in its $S U(N)$ indices. It is easy to see that in the normal frame these equations imply:

$$
\begin{aligned}
\left.M_{A D M}\right|_{f i x} & \equiv\left|Z_{1}\right| \neq 0 \\
\left|Z_{i}\right| & =0 \quad(i=2, \cdots, N / 2)
\end{aligned}
$$

The main purpose of this section is to provide particular expressions which give the entropy formula as a moduli-independent quantity in the entire moduli space and not just at the critical points. Namely, we are looking for quantities $S\left(Z_{A B}(\phi), \bar{Z}^{A B}(\phi), Z_{I}(\phi), \bar{Z}^{I}(\phi)\right)$ such that $\frac{\partial}{\partial \phi^{i}} S=0, \phi^{i}$ being the moduli coordinates.

These formulae generalize the quartic $E_{7(-7)}$ invariant of $N=8$ supergravity [58] to all other cases.

Let us first consider the theories $N=3,4$, where matter can be present [47], 63].

The U-duality groups are, in these cases, $S U(3, n)$ and $S U(1,1) \times S O(6, n)$ respectively (Here we denote by U-duality group the isometry group $G$ acting on the scalars, although only a restriction of it to integers is the proper U-duality group [16]). The central and matter charges $Z_{A B}, Z_{I}$ transform in an obvious way under the isotropy groups

$$
\begin{aligned}
& H=S U(3) \times S U(n) \times U(1) \quad(N=3) \\
& H=S U(4) \times O(n) \times U(1) \quad(N=4)
\end{aligned}
$$

Under the action of the elements of $G / H$ the charges get mixed with their complex conjugate.

For $N=3$ :

$$
\begin{aligned}
P^{A B C D} & =P_{I J}=0, P_{A B I} \equiv \epsilon_{A B C} P_{I}^{C} \\
Z_{A B} & \equiv \epsilon_{A B C} Z^{C}
\end{aligned}
$$


Then the variations are:

$$
\begin{aligned}
\delta Z^{A} & =\xi_{I}^{A} \bar{Z}^{I} \\
\delta Z_{I} & =\xi_{I}^{A} \bar{Z}_{A}
\end{aligned}
$$

where $\xi_{I}^{A}$ are infinitesimal parameters of $K=G / H$. Indeed, once the covariant derivatives are known, the variations are obtained by the substitution $\nabla \rightarrow \delta, P \rightarrow \xi$.

With a simple calculation, the U-invariant expression is:

$$
S=Z^{A} \bar{Z}_{A}-Z_{I} \bar{Z}^{I}
$$

In other words, $\nabla_{i} S=\partial_{i} S=0$, where the covariant derivative is defined in ref. [20].

Note that at the attractor point $\left(Z_{I}=0\right)$ it coincides with the moduli-dependent potential (6.88) computed at its extremum.

For $N=4$

$$
\begin{aligned}
P_{A B C D} & =\epsilon_{A B C D} P, \quad P_{I J}=\eta_{I J} \bar{P} \\
P_{A B I} & =\frac{1}{2} \eta_{I J} \epsilon_{A B C D} \bar{P}^{C D J}
\end{aligned}
$$

and the transformations of $K=\frac{S U(1,1)}{U(1)} \times \frac{O(6, n)}{O(6) \times O(n)}$ are:

$$
\begin{aligned}
\delta Z_{A B} & =\frac{1}{2} \xi \epsilon_{A B C D} \bar{Z}^{C D}+\xi_{A B I} \bar{Z}^{I} \\
\delta Z_{I} & =\bar{\xi} \eta_{I J} \bar{Z}^{J}+\frac{1}{2} \xi_{A B I} \bar{Z}^{A B}
\end{aligned}
$$

with $\bar{\xi}^{A B I}=\frac{1}{2} \eta^{I J} \epsilon^{A B C D} \xi_{C D J}$.

There are three $O(6, n)$ invariants given by $I_{1}, I_{2}, \bar{I}_{2}$ where:

$$
\begin{aligned}
& I_{1}=\frac{1}{2} Z_{A B} \bar{Z}_{A B}-Z_{I} \bar{Z}^{I} \\
& I_{2}=\frac{1}{4} \epsilon^{A B C D} Z_{A B} Z_{C D}-\bar{Z}_{I} \bar{Z}^{I}
\end{aligned}
$$

and the unique $S U(1,1) \times O(6, n)$ invariant $S, \nabla S=0$, is given by:

$$
S=\sqrt{\left(I_{1}\right)^{2}-\left|I_{2}\right|^{2}}
$$

At the attractor point $Z_{I}=0$ and $\epsilon^{A B C D} Z_{A B} Z_{C D}=0$ so that $S$ reduces to the square of the BPS mass.

For $N=5,6,8$ the U-duality invariant expression $S$ is the square root of a unique invariant under the corresponding U-duality groups $S U(5,1), O^{*}(12)$ and $E_{7(-7)}$. The strategy is to find a quartic expression $S^{2}$ in terms of $Z_{A B}$ such that $\nabla S=0$, i.e. $S$ is moduli-independent.

As before, this quantity is a particular combination of the $H$ quartic invariants.

For $S U(5,1)$ there are only two $U(5)$ quartic invariants. In terms of the matrix $A_{A}^{B}=$ $Z_{A C} \bar{Z}^{C B}$ they are: $(\operatorname{Tr} A)^{2}, \operatorname{Tr}\left(A^{2}\right)$, where

$$
\begin{aligned}
\operatorname{Tr} A & =Z_{A B} \bar{Z}^{B A} \\
\operatorname{Tr}\left(A^{2}\right) & =Z_{A B} \bar{Z}^{B C} Z_{C D} \bar{Z}^{D A}
\end{aligned}
$$


As before, the relative coefficient is fixed by the transformation properties of $Z_{A B}$ under $\frac{S U(5,1)}{U(5)}$ elements of infinitesimal parameter $\xi^{C}$ :

$$
\delta Z_{A B}=\frac{1}{2} \xi^{C} \epsilon_{C A B P Q} \bar{Z}^{P Q}
$$

It then follows that the required invariant is:

$$
S=\frac{1}{2} \sqrt{4 \operatorname{Tr}\left(A^{2}\right)-(\operatorname{Tr} A)^{2}}
$$

For $N=8$ the $S U(8)$ invariants are:

$$
\begin{aligned}
I_{1} & =(\operatorname{Tr} A)^{2} \\
I_{2} & =\operatorname{Tr}\left(A^{2}\right) \\
I_{3} & =\operatorname{Pf} Z \\
& =\frac{1}{2^{4} 4 !} \epsilon^{A B C D E F G H} Z_{A B} Z_{C D} Z_{E F} Z_{G H}
\end{aligned}
$$

The $\frac{E_{7(-7)}}{S U(8)}$ transformations are:

$$
\delta Z_{A B}=\frac{1}{2} \xi_{A B C D} \bar{Z}^{C D}
$$

where $\xi_{A B C D}$ satisfies the reality constraint:

$$
\xi_{A B C D}=\frac{1}{24} \epsilon_{A B C D E F G H} \bar{\xi}^{E F G H}
$$

One finds the following $E_{7(-7)}$ invariant [58]:

$$
S=\frac{1}{2} \sqrt{4 T r\left(A^{2}\right)-(\operatorname{Tr} A)^{2}+32 \operatorname{Re}(\operatorname{Pf} Z)}
$$

The $N=6$ case is the more complicated because under $U(6)$ the left-handed spinor of $O^{*}(12)$ splits into:

$$
32_{L} \rightarrow(15,1)+(\overline{15},-1)+(1,-3)+(1,3)
$$

The transformations of $\frac{O^{*}(12)}{U(6)}$ are:

$$
\begin{aligned}
\delta Z_{A B} & =\frac{1}{4} \epsilon_{A B C D E F} \xi^{C D} \bar{Z}^{E F}+\xi_{A B} \bar{X} \\
\delta X & =\frac{1}{2} \xi_{A B} \bar{Z}^{A B}
\end{aligned}
$$

where we denote by $X$ the $S U(6)$ singlet. The quartic $U(6)$ invariants are:

$$
\begin{aligned}
I_{1} & =(\operatorname{Tr} A)^{2} \\
I_{2} & =\operatorname{Tr}\left(A^{2}\right) \\
I_{3} & =\operatorname{Re}(\operatorname{Pf} Z X) \\
& =\frac{1}{2^{3} 3 !} \operatorname{Re}\left(\epsilon^{A B C D E F} Z_{A B} Z_{C D} Z_{E F} X\right) \\
I_{4} & =(\operatorname{Tr} A) X \bar{X} \\
I_{5} & =X^{2} \bar{X}^{2}
\end{aligned}
$$


The unique $O^{*}(12)$ invariant is:

$$
\begin{aligned}
S & =\frac{1}{2} \sqrt{4 I_{2}-I_{1}+32 I_{3}+4 I_{4}+4 I_{5}} \\
\nabla S & =0
\end{aligned}
$$

Note that at the attractor point $\operatorname{Pf} Z=0, X=0$ and $S$ reduces to the square of the BPS mass.

We note that most of the results given above can be obtained in a simple way by performing the relevant computations in the so called "normal frame" of the $Z_{A B}$ matrix.

Indeed, in order to determine the quartic U-invariant expressions $S^{2}, \nabla S=0$, of the $N>4$ theories, it is useful to use, as a calculational tool, transformations of the coset which preserve the normal form of the $Z_{A B}$ matrix. It turns out that these transformations are certain Cartan elements in $K=G / H$ [30], that is they belong to $O(1,1)^{p} \in K$, with $p=1$ for $N=5, p=3$ for $N=6,8$.

These elements act only on the $Z_{A B}$ (in normal form), but they uniquely determine the U-invariants since they mix the eigenvalues $e_{i}(i=1, \cdots,[N / 2])$.

For $N=5, S U(5,1) / U(5)$ has rank one (see ref. [64]) and the element is:

$$
\delta e_{1}=\xi e_{2} ; \quad \delta e_{2}=\xi e_{1}
$$

which is indeed a $O(1,1)$ transformation with unique invariant

$$
\left|\left(e_{1}\right)^{2}-\left(e_{2}\right)^{2}\right|=\frac{1}{2} \sqrt{8\left(\left(e_{1}\right)^{4}+\left(e_{2}\right)^{4}\right)-4\left(\left(e_{1}\right)^{2}+\left(e_{2}\right)^{2}\right)^{2}}
$$

For $N=6$, we have $\xi_{1} \equiv \xi_{12} ; \xi_{2} \equiv \xi_{34} ; \xi_{3} \equiv \xi_{56}$ and we obtain the 3 Cartan elements of $O^{*}(12) / U(6)$, which has rank 3 , that is it is a $O(1,1)^{3}$ in $O^{*}(12) / U(6)$. Denoting by $e$ the singlet charge, we have the following $O(1,1)^{3}$ transformations:

$$
\begin{aligned}
\delta e_{1} & =\xi_{2} e_{3}+\xi_{3} e_{2}+\xi_{1} e \\
\delta e_{2} & =\xi_{1} e_{3}+\xi_{3} e_{1}+\xi_{2} e \\
\delta e_{3} & =\xi_{1} e_{2}+\xi_{2} e_{1}+\xi_{3} e \\
\delta e & =\xi_{1} e_{1}+\xi_{2} e_{2}+\xi_{3} e_{3}
\end{aligned}
$$

these transformations fix uniquely the $O^{*}(12)$ invariant constructed out of the five $U(6)$ invariants displayed in (6.116-6.120). For $N=8$ the infinitesimal parameter is $\xi_{A B C D}$ and, using the reality condition, we get again a $O(1,1)^{3}$ in $E_{7(-7)} / S U(8)$. Setting $\xi_{1234}=$ $\xi_{5678} \equiv \xi_{12}, \xi_{1256}=\xi_{3478} \equiv \xi_{13}, \xi_{1278}=\xi_{3456} \equiv \xi_{14}$, we have the following set of transformations:

$$
\begin{aligned}
\delta e_{1} & =\xi_{12} e_{2}+\xi_{13} e_{3}+\xi_{14} e_{4} \\
\delta e_{2} & =\xi_{12} e_{1}+\xi_{13} e_{4}+\xi_{14} e_{3} \\
\delta e_{3} & =\xi_{12} e_{4}+\xi_{13} e_{1}+\xi_{14} e_{2} \\
\delta e_{4} & =\xi_{12} e_{3}+\xi_{13} e_{2}+\xi_{14} e_{1}
\end{aligned}
$$

These transformations fix uniquely the relative coefficients of the three $S U(8)$ invariants:

$$
\begin{aligned}
& I_{1}=4\left(e_{1}^{2}+e_{2}^{2}+e_{3}^{2}+e_{4}^{2}\right)^{2} \\
& I_{2}=2\left(e_{1}^{4}+e_{2}^{4}+e_{3}^{4}+e_{4}^{4}\right) \\
& I_{3}=e_{1} e_{2} e_{3} e_{4}
\end{aligned}
$$


It is easy to see that the transformations (6.125-6.128) and (6.1296.132) correspond to three commuting matrices (with square equal to $\mathbb{1}$ ):

$$
\left(\begin{array}{llll}
0 & 0 & 0 & 1 \\
0 & 0 & 1 & 0 \\
0 & 1 & 0 & 0 \\
1 & 0 & 0 & 0
\end{array}\right) ;\left(\begin{array}{llll}
0 & 1 & 0 & 0 \\
1 & 0 & 0 & 0 \\
0 & 0 & 0 & 1 \\
0 & 0 & 1 & 0
\end{array}\right) ;\left(\begin{array}{llll}
0 & 0 & 1 & 0 \\
0 & 0 & 0 & 1 \\
1 & 0 & 0 & 0 \\
0 & 1 & 0 & 0
\end{array}\right)
$$

which are proper non compact Cartan elements of $K$. The reason we get the same transformations for $N=6$ and $N=8$ is because the extra singlet $e$ of $N=6$ can be identified with the fourth eigenvalue of the central charge of $N=8$.

\section{Extrema of the BPS mass and fixed scalars}

In this section we would like to extend the analysis of the extrema of the black-hole induced potential

$$
V=\frac{1}{2} Z_{A B} \bar{Z}^{A B}+Z_{I} \bar{Z}^{I}
$$

which was performed in ref [57] for the $N=2$ case to all $N>2$ theories. We recall that, in the case of $N=2$ special geometry with metric $g_{i \bar{\jmath}}$, at the fixed scalar critical point $\partial_{i} V=0$ the Hessian matrix reduces to:

$$
\begin{aligned}
\left(\nabla_{i} \nabla_{\bar{\jmath}} V\right)_{\text {fixed }} & =\left(\partial_{i} \partial_{\bar{\jmath}} V\right)_{\text {fixed }}=2 g_{i \bar{\jmath}} V_{\text {fixed }} \\
\left(\nabla_{i} \nabla_{j} V\right)_{\text {fixed }} & =0
\end{aligned}
$$

The Hessian matrix is strictly positive-definite if the critical point is not at the singular point of the vector multiplet moduli-space. This matrix was related to the Weinhold metric earlier introduced in the geometric approach to thermodynamics and used for the study of critical phenomena [57]. For $N$-extended supersymmetry, a form of this matrix was also given and shown to be equal to

$$
\begin{aligned}
V_{i j} & =\left(\partial_{i} \partial_{j} V\right)_{\text {fixed }} \\
& =Z_{C D} Z^{A B}\left(\frac{1}{2} P_{, j}^{C D P Q} P_{A B P Q, i}\right. \\
& \left.+P_{I, j}^{C D} P_{A B, j}^{I}\right) .
\end{aligned}
$$

It is our purpose to further investigate properties of the Weinhold metric for fixed scalars. Let us first observe that the extremum conditions $\nabla_{i} V=0$, using the relation between the covariant derivatives of the central charges, reduce to the conditions:

$$
\epsilon^{A B C D L_{1} \cdots L_{N-4}} Z_{A B} Z_{C D}=0, \quad Z_{I}=0
$$

These equations give the fixed scalars in terms of electric and magnetic charges and also show that the topological invariants of the previous section reduce to the extremum of the

\footnotetext{
${ }^{6}$ Generically the indices $i, j$ refer to real coordinates, unless the manifold is Kählerian, in which case we use holomorphic coordinates and formula (7.141) reduces to the hermitean $i \bar{\jmath}$ entries of the Hessian matrix.
} 
square of the ADM mass since, when the above conditions are fulfilled, $(\operatorname{Tr} A)^{2}=2 \operatorname{Tr}\left(A^{2}\right)$, where $A_{A}^{B}=Z_{A B} \bar{Z}^{B C}$. On the other hand, when these conditions are fulfilled, it is easy to see that the Hessian matrix is degenerate. To see this, it is sufficient to go, making an $H$ transformation, to the normal frame in which these conditions imply $Z_{12} \neq 0$ with the other charges vanishing. Then we have:

$$
\begin{aligned}
& \left.\partial_{i} \partial_{j} V\right|_{\text {fixed }}= \\
& 4\left|Z_{12}\right|^{2}\left(\frac{1}{2} P_{j}^{12 a b} P_{12 a b, i}+P_{, j}^{12 I} P_{12 I, i}\right),
\end{aligned}
$$

where $a, b \neq 1,2$. To understand the pattern of degeneracy for all $N$, we observe that when only one central charge is not vanishing the theory effectively reduces to an $N=2$ theory. Then the actual degeneracy respects $N=2$ multiplicity of the scalars degrees of freedom in the sense that the degenerate directions will correspond to the hypermultiplet content of $N>2$ theories when decomposed with respect to $N=2$ supersymmetry.

Note that for $N=3, N=4$, where $P_{A B I}$ is present, the Hessian is block diagonal. For $N=3$, referring to eq. (6.94), since the scalar manifold is Kähler, $P_{A B I}$ is a $(1,0)$-form while $P^{A B I}=\bar{P}_{A B I}$ is a $(0,1)$-form.

The scalars appearing in the $N=2$ vector multiplet and hypermultiplet content of the vielbein are $P_{3 I}$ for the vector multiplets and $P_{a I}(a=1,2)$ for the hypermultiplets. From equation (7.143), which for the $N=3$ case reads

$$
\left.\partial_{\bar{\jmath}} \partial_{i} V\right|_{\text {fixed }}=2\left|Z_{12}\right|^{2} P_{3 I, \bar{\jmath}} P_{, i}^{3 I}
$$

we see that the metric has $4 n$ real directions corresponding to $n$ hypermultiplets which are degenerate.

For $N=4$, referring to (6.98), $P$ is the $S U(1,1) / U(1)$ vielbein which gives one matter vector multiplet scalar while $P_{12 I}$ gives $n$ matter vector multiplets. The directions which are hypermultiplets correspond to $P_{1 a I}, P_{2 a I}(a=3,4)$. Therefore the "metric" $V_{i j}$ is of rank $2 n+2$.

For $N>4$, all the scalars are in the gravity multiplet and correspond to $P_{A B C D}$.

The splitting in vector and hypermultiplet scalars proceeds as before. Namely, in the $N=5$ case we set $P_{A B C D}=\epsilon_{A B C D L} P^{L}(A, B, C, D, L=1, \cdots 5)$. In this case the vector multiplet scalars are $P^{a}(a=3,4,5)$ while the hypermultiplet scalars are $P^{1}, P^{2}\left(n_{V}=3\right.$, $\left.n_{h}=1\right)$.

For $N=6$, we set $P_{A B C D}=\frac{1}{2} \epsilon_{A B C D E F} P^{E F}$. The vector multiplet scalars are now described by $P^{12}, P^{a b}(A, B, \ldots=1, \ldots, 6 ; a, b=3, \cdots 6)$, while the hypermultiplet scalars are given in terms of $P^{1 a}, P^{2 a}$. Therefore we get $n_{V}=6+1=7, n_{h}=4$.

This case is different from the others because, besides the hypermultiplets $P^{1 a}, P^{2 a}$, also the vector multiplet direction $P^{12}$ is degenerate. Finally, for $N=8$ we have $P_{1 a b c}, P_{2 a b c}$ as hypermultiplet scalars and $P_{a b c d}$ as vector multiplet scalars, which give $n_{V}=15, n_{h}=10$ (note that in this case the vielbein satisfies a reality condition: $\left.P_{A B C D}=\frac{1}{4 !} \epsilon_{A B C D P Q R S} \bar{P}^{P Q R S}\right)$. We have in this case 40 degenerate directions.

In conclusion we see that the rank of the matrix $V_{i j}$ is $(N-2)(N-3)+2 n$ for all the four dimensional theories. 


\section{Relations to string theories}

$N$-extended supergravities are related to strings compactified on six-manifolds $M_{N}$ preserving $N$ supersymmetries at $D=4$. Since we are presently considering $N>2$, the most common cases are $N=4$ and $N=8$. The first can be achieved in heterotic or Type II string, with $M_{4}=T_{6}$ in heterotic and $M_{4}=K_{3} \times T_{2}$ in Type II theory. These theories are known to be dual at a non perturbative level [16], [2], [31]. $N=8$ corresponds to $M_{8}=T_{6}$ in Type II.

Less familiar are the $N=3,5$ and 6 cases which were studied in ref. 655.

Interestingly enough, the latter cases can be obtained by compactification of Type II on asymmetric orbifolds with $3=2_{L}+1_{R}, 5=4_{L}+1_{R}$ and $6=4_{L}+2_{R}$ respectively.

BPS states considered in this paper should correspond to massive states in these theories for which only a subset of them is known in the perturbative framework.

In attemps to test non perturbative string properties it would be interesting to check the existence of the BPS states and their entropy by using microscopic considerations.

We finally observe that, unlike $N=8$, the moduli spaces of $N=3,5,6$ theories are locally Kählerian (as $N=2$ ) with coset spaces of rank $3(n \geq 3), 1$ and 3 respectively.

For $N=5,6$ these spaces are also special Kähler (which is also the case for $N=3$ when $n=1,3)$ 66 667.

We can use the previous observations to construct U-invariants for some $N=2$ special geometries looking at the representation content of vectors and their duals with respect to U-dualities. Let us first consider $N=2$ theories with U-duality $S U(1, n)$ and $S U(3,3)$. These groups emerge in discussing string compactifications on some $N=2$ orbifolds (i.e. orbifold points of Calabi-Yau threefolds) 67 68. The vector content is respectively given by the fundamental representation of $S U(1, n)$ and the twenty dimentional threefold antisymmetric rep. of $S U(3,3)$ 69. Amazingly, the first representation occurs as in $N=3$ matter coupled theories, while the latter is the same as in $N=5$ supergravity (note that $S U(1, n), S U(3, n)$ and $S U(3,3), S U(5,1)$ are just different non compact forms of the same $S U(m)$ groups). From the results of the previous section we conclude that the special manifolds $\frac{S U(1, n)}{S U(n) \times U(1)}$ and $\frac{S U(3,3)}{S U(3) \times S U(3) \times U(1)}$ admit respectively a quadratic 60, 700 and a quartic topological invariant. The $N=2$ special manifold $\frac{O^{*}(12)}{U(6)}$ has a vector content which is a left spinor of $O^{*}(12)$, as in the $N=6$ theory, therefore it admits a

quartic invariant. Finally, the $N=2$ special manifolds $\frac{S U(1,1)}{U(1)} \times \frac{O(2, n)}{O(2) \times O(n)}$, which emerge in $N=2$ compactifications of both heterotic and Type II strings 68, admit a quartic invariant which can be read from the $N=4$ quartic invariant in which the $\frac{S U(1,1)}{U(1)}$ matter charge is identified with the second eigenvalue of the $N=4$ central charge. All the above topological invariants can then be interpreted as entropy of a variety of $N=2$ black-holes.

\section{References}

[1] For recent reviews see: J. Schwarz, preprint CALTECH-68-2065, hep-th/9607021; M. Duff, preprint CPT-TAMU-33-96, hep-th/9608117; A. Sen, preprint MRI-PHY96-28, hep-th/9609176; J. Polchinski, hep-th/9607050

[2] E. Witten, "String Theory Dynamics in Various Dimensions", hep-th/9503124, Nucl. Phys. B 443 (1995) 85 
[3] C. Vafa, "Evidence for F-Theory", hep-th/9602022

[4] C. M. Hull and P. K. Townsend, Nucl. Phys. B 451 (1995) 525, hep-th/9505073

[5] E. Witten, "Some Comments on String Dynamics", hep-th/9507121, Contributed to STRINGS '96: Future Perspectives in Sttring Theory, Los Angeles, California, 13-18 March 1995; N. Seiberg and E. Witten, hep-th/9603003, Nucl. Phys. B 471 (1996) 121

[6] A. Strominger, Nucl. Phys. B 451 (1995) 96, hep-th/9504090; B. Greene, D. Morrison and A. Strominger, Nucl. Phys. B 451 (1995) 109, hep-th/9504145.

[7] H. Ooguri and C. Vafa, "Summing Up D-Instantons", hep-th/9608079; N. Seiberg and S. Shenker, "Hypermultiplets Moduli Space and String Compactification to Three Dimensions", hep-th/9608086

[8] D. R. Morrison and C. Vafa, Nucl. Phys. B 473 (1996) 74, hep-th/9602114; D. R. Morrison and C. Vafa, Nucl. Phys. B 476 (1996) 437, hep-th/9603161

[9] E. Witten, hep-th/9603150, Nucl. Phys. B 471 (1996) 195

[10] for a recent review see: J. Maldacena, "Black Holes in String Theory", hepth/9607235

[11] A. Salam and E. Sezgin, "Supergravities in diverse Dimensions" Edited by A. Salam and E. Sezgin, North-Holland, World Scientific 1989, vol. 1

[12] R. Haag, J. T. Lopuszanski and M Sohnius, "All Possible Generators of Supersymmetries of the S Matrix" Nucl. Phys. B 88 (1975) 257

[13] P. K. Townsend, "p-Brane Democracy", hep-th/9507048, to appear in the Proceedings of the March '95 PASCOS/Johns Hopking conference

[14] E. Cremmer, J. Scherk and S. Ferrara, Phys. Lett. 74 B (1978) 61

[15] S. Ferrara, J. Scherk and B. Zumino, Nucl. Phys. B 121 (1977) 393

[16] C. M. Hull and P. K. Townsend, Nucl. Phys. B451 (1995) 525, hep-th/9505073

[17] S. Ferrara, C. Savoy and B. Zumino, Phys. Lett. 100 B (1981) 393

[18] A. Strominger, Comm. Math. Phys. 133 (1990) 163

[19] A. Ceresole, R. D’Auria, S. Ferrara, W. Lerche and J. Louis, Int. Jour. Mod. Phys. A Vol. 8 (1993) 79

[20] L. Andrianopoli, R. D'Auria and S. Ferrara, hep-th/9612105

[21] L. Andrianopoli, R. D'Auria and S. Ferrara, hep-th/9703156

[22] S. Ferrara, R. Kallosh and A. Strominger, Phys. Rev. D52 (1995) 5412, hepth/9508072; A. Strominger, Phys. Lett. B383 (1996) 39, hep-th/9602111 
[23] S. Ferrara and R. Kallosh, Phys. Rev. D54 (1996) 1514, hep-th/9602136

[24] S. Ferrara and R. Kallosh, Phys. Rev. D54 (1996) 1525, hep-th/9603090

[25] J. Schwarz, "Lectures on Superstring and M-theory Dualities", hep-th/9607201

[26] M. Dine, P. Huet and N. Seiberg, Nucl. Phys. B 322 (1989) 301

[27] A. C. Cadavid, A. Ceresole, R. D'Auria and S. Ferrara, hep-th/9506144, Phys. Lett. B 357 (1995) 76; S. Ferrara, R. Khuri and R. Minasian, hep-th/9602102, Phys. Lett. B 375 (1996) 81; I. Antoniadis, S. Ferrara and T. R. Taylor, Nucl. Phys. B 460 (1996) 489

[28] A. Kumar and C. Vafa, "U-Manifolds", hep-th/9611007

[29] E. Cremmer, in "Supergravity '81", ed. by S. Ferrara and J. G. Taylor, pag. 313; B. Julia in "Superspace \&S Supergravity" ed. by S. W. Hawking and M. Rocek, Cambridge (1981) pag. 331

[30] L. Andrianopoli, R. D'Auria, S. Ferrara, P. Fré and M. Trigiante, " $R$ - $R$ Scalars, U-Duality and Solvable Lie Algebras", hep-th/9611014

[31] M. J. Duff, J. T. Liu and J. Rahmfeld, "Four dimensional string/string/string triality”, Nucl. Phys. B459 (1996) 125, hep-th/9508094

[32] A. Klemm, W. Lerche and P. Mayr, Phys. Lett. B 357 (1995) 313

[33] A. Ceresole, R. D’Auria, S. Ferrara and A. Van Proyen, Nucl. Phys. B 444 (1995) 92

[34] S. Ferrara, J. A. Harvey, A. Strominger and C. Vafa, Phys. Lett. B 361 (1995) 59, hep-th/9505162.

[35] S. Kachru and C. Vafa, hep-th/9505105, Nucl. Phys. B 450 (1995) 69,

[36] S. Ferrara, R. Minasian and A. Sagnotti, "Low Energy Analysis of $M$ and F Theories on Calabi Yau Manifolds", hep-th/9604097

[37] A. Sagnotti, Non Perturbative Quantum Field Theory, Cargese Lectures Ed. by G. Mack et al. (Pergamon Press 1988) 521; J. Dai, R. G. Leigh and J. Polchinski, Mod. Phys. Lett. A 4 (1989) 2073

[38] J. Polchinski, hep-th/9510017 Phys. Rev. Lett 75 (1995) 4724; J. Polchinski, S. Chaudhuri and C. Johnson, Notes on D-Branes, hep-th/9602052

[39] L. Castellani, R. D'Auria and P. Fré, "Supergravity and Superstrings: a Geometric Perspective" World Scientific 1991

[40] R. D'Auria and P. Fre', Nucl. Phys. B201 (1982) 101

[41] J.V. van Holten and A. Van Proeyen, J. Phys. A; Math. Gen. 15 (1982) 3763 
[42] J. A. de Azcarraga, J. P. Gauntlett, J. M. Izquierdo and P. K. Townsend, Phys. Rev. Lett. 189B (1989) 2443

[43] I. Bars, hep-th/9604139, Phys. Rev. D 54 (1996) 5203; I. Bars, "Algebraic Structure of $S$-Theory" hep-th/9608061

[44] A. Ceresole, R. D'Auria and S. Ferrara, hep-th/9509160, "S-Duality and Mirror Symmetry" Nucl. Phys. (Proc. Supplements) B 46 (1996) 67, Eds. E. Gava, K. S. Narain, C. Vafa

[45] M. K. Gaillard and B. Zumino, Nucl. Phys. B 193 (1981) 221

[46] S. Cecotti, S. Ferrara and L. Girardello, Nucl. Phys. B 308 (1988) 436

[47] L. Castellani, A. Ceresole, R. D'Auria, S. Ferrara. P. Fré and E. Maina, Nucl. Phys. B 286 (1986) 317

[48] E. Bergshoeff, I. G. Koh and E. Sezgin, Phys. Lett. 155 B (1985) 71; M. de Roo and F. Wagemans, Nucl. Phys. B 262 (1985) 644

[49] S. Ferrara and A. Strominger, " $N=2$ Space-Time supersymmetry and Calabi Yau Moduli Space", in Proceedings of College Station Workshop "Strings '89', pag. 245, eds. Arnowitt et al., World scientific 1989; P. Candelas and X. C. de la Ossa, Nucl. Phys. B 355 (1991) 455; B. de Wit and A. Van Proeyen, nucl. phys. B 245 (1984) 89; E. Cremmer, C. Kounnas, A. Van Proeyen, J. P. Derendinger, S. Ferrara, B. de Wit and L. Girardello, Nucl. Phys. B 250 (1985) 385; B. de Wit, P. G. Lawers and A. Van Proeyen, Nucl. Phys. B 255 (1985) 569; S. Ferrara, C. Kounnas, D. Lüst and F. Zwirner, Nucl. Phys. B 365 (1991) 431; L. Castellani, R. D'Auria and S. Ferrara, Class. Quant. Grav. 7 (1990) 1767; L. Castellani, R. D'Auria and S. Ferrara, Phys. Lett. 241 B (1990) 57

[50] E. Cremmer and A. Van Proeyen, Class. quant. Grav. 2 (1985) 445

[51] B. de Wit and H. Nicolai, Nucl. Phys. B 188 (1981) 98

[52] E. Cremmer and B. Julia, Phys. Lett. 80 B (1978) 48

[53] For a review, see for instance: M. J. Duff, R. R. Khuri and J. X. Lu, String solitons, Phys. Rep. 259 (1995) 213; M. J. Duff, Kaluza-Klein theory in perspective, in Proceedings of the Nobel Symposium Oskar Klein Centenary, Stockholm, September 1994 (World Scientific, 1995), E. Lindstrom editor, hep-th/9410046; G. Horowitz, UCSBTH-96-07, gr-qc/9604051; J. M. Maldacena, Ph.D. thesis, hep-th/9607235; M. Cvetic, UPR-714-T, hep-th/9701152

[54] G. Gibbons, in Unified theories of Elementary Particles. Critical Assessment and Prospects, Proceedings of the Heisemberg Symposium, München, West Germany, 1981, ed. by P. Breitenlohner and H. P. Dürr, Lecture Notes in Physics Vol. 160 (Springer-Verlag, Berlin, 1982); G. W. Gibbons and C. M. Hull, Phys. lett. 109B (1982) 190; G. W. Gibbons, in Supersymmetry, Supergravity and Related Topics, Proceedings of the XVth GIFT International Physics, Girona, Spain, 1984, ed. by 
F. del Aguila, J. de Azcárraga and L. Ibáñez, (World Scientific, 1995), pag. 147; R. Kallosh, A. Linde, T. Ortin, A. Peet and A. Van Proeyen, Phys. Rev. D46 (1992) 5278; R. Kallosh, T. Ortin and A. Peet, Phys. Rev. D47 (1993) 5400; R. Kallosh, Phys. Lett. B282 (1992) 80; R. Kallosh and A. Peet, Phys. Rev. D46 (1992) 5223; A. Sen, Nucl. Phys. B440 (1995) 421; Phys. Lett. B303 (1993) 221; Mod. Phys. Lett. A10 (1995) 2081; J. Schwarz and A. Sen, Phys. Lett. B312 (1993) 105; M. Cvetic and D. Youm, Phys. Rev. D53 (1996) 584; M. Cvetic and A. A. Tseytlin, Phys. Rev. D53 (1996) 5619; M. Cvetic and C. M. Hull, Nucl. Phys. B480 (1996) 296

[55] A. Strominger and C. Vafa, Phys. Lett. B379 (1996) 99, hep-th/9601029; C. G. Callan and J. M. Maldacena, Nucl. Phys. B472 (1996) 591, hep-th/9602043; G. Horowitz and A. Strominger, Phys. Rev. Lett. B383 (1996) 2368, hep-th/9602051; R. Dijkgraaf, E. Verlinde, H. Verlinde, Nucl.Phys. B486 (1997) 77, hep-th/9603126; P. M. Kaplan, D. A. Lowe, J. M. Maldacena and A. Strominger, hep-th/9609204; J. M. Maldacena, hep-th/9611163

[56] L. Susskind, hep-th/9309145; L. Susskind and J. Uglum, Phys. Rev. D50 (1994) 2700; F. Larsen and F. Wilczek, Phys. Lett. B375 (1996) 37, hep-th/9511064

[57] S. Ferrara, G. W. Gibbons and R. Kallosh, hep-th/9702103

[58] R. Kallosh and B. Kol, Phys. Rev. D53 (1996) 5344

[59] S. Ferrara, C. Savoy and B. Zumino, Phys. Lett. 100B (1981) 393

[60] A. Ceresole, R. D'Auria and S. Ferrara, in "S-Duality and Mirror symmetry", Nucl. Phys. (Proc. Suppl.) B46 (1996) 67, ed. E. Gava, K. S. Narain and C. Vafa, hepth/9509160

[61] L. Andrianopoli, R. D'Auria and S. Ferrara, "Central Extension of Extended Supergravities in Diverse Dimensions", hep-th/9608015, to appear in Int. Jour. Mod. Phys. A

[62] P. Breitenlohner, D. Maison and G. W. Gibbons, Commun. Math. Phys. 120 (1988) 295; G. W. Gibbons, R. Kallosh and B. Kol, Phys. Rev. Lett. 77 (1996) 4992, hepth/9607108

[63] E. Bergshoeff, I. G. Koh and E. Sezgin, Phys. Lett. 155B (1985) 71; M. de Roo and F. Wagemans, nucl. Phys. B262 (1985) 644

[64] R. Gilmore, "Lie groups, Lie algebras and some of their applications", (1974) ed. J. Wiley and sons

[65] S. Ferrara and C. Kounnas, Nucl. Phys. B328 (1989) 406; S. Ferrara and P. Fré, Int. Jour. Mod. Phys. A Vol. 5, No. 5 (1990) 989

[66] E. Cremmer and A. Van Proeyen, Class. Quant. Grav. 2 (1985) 445

[67] S. Cecotti, S. Ferrara and L. Girardello, Int. Jour. Mod. Phys. A Vol. 4 (1989) 2475 
[68] N. Seiberg, Nucl. Phys. B303 (1988) 286; L. Dixon, V. Kaplunowsky, J. Louis, Nucl. Phys. B329 (1990) 27; S. Ferrara, C. Kounnas and M. Porrati, Phys. Lett. B181 (1986) 26

[69] S. Ferrara, P. Fré and P. Soriani, Class. Quantum Grav. 9 (1992) 1649

[70] K. Behrndt, W. A. Sabra, hep-th/9702010 